\title{
Ergebnisse und Fazit der Qualia-Revision
}

\subsection{Eine längst fällige Revision und die Verwirrung über das Explanandum}

Diese Arbeit soll als Beitrag für eine längst fällige Qualia-Revision gelten. Warum sind eine Qualia-Revision und eine damit verbundene Kritik von angeblichen, aber phänomenal inadäquaten Qualia-Eigenschaften (bzw. eine Kritik von unhaltbaren Qualia-Begriffen) so wichtig?

Nur mit einem phänomenal adäquaten Qualia-Begriff lässt sich das Explanandum des hard problem verstehen. Das Desiderat eines phänomenal adäquaten Qualia-Begriffs ist folglich unverzichtbar, um überhaupt erst beginnen zu können, sich mit dem hard problem auseinander zu setzen. Denn wie soll man sich an ein Problem wagen, wenn dessen Explanandum gar nicht genau eruiert worden ist? Zu welcher Verwirrung es führt, wenn nicht klar ist, worin das Explanandum des hard problem besteht, hat die Analyse in Abschnitt 8.1 gezeigt. Weil bspw. Dennett der Frage, was unter Phänomenalität verstanden werden soll, nur eine halbe Seite widmet, offenbaren sich seine fünfzehn detailliert ausgearbeiteten $>$ Intuitionspumpen ${ }^{1}$ und seine darauf aufbauenden Argumente, dass es keine Qualia >gibt<, unter der Lupe phänomenaler Adäquatheit als Strohmann-Argumente: Dennett hat ge-

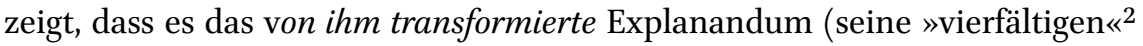
Qualia) nicht gibt. Weil der bisherige, insbesondere von reduktionistischer Seite verwendete Qualia-Begriff gegenüber der Heterogenität, Reichhaltigkeit, Feinkörnigkeit und Ubiquität des Phänomens nicht differenziert genug ist, erweist sich auch das hard problem als nicht adäquat erfasst und in seiner Bedeutung als erheblich unterschätzt. Denn Qualia stellen keinesfalls irgendwelche »marginalen ${ }^{3}$ Phänomene mit Eigenschaften dar, die nur auf eine eigentümliche und im Hinblick auf das hard problem ohne Schwierigkeiten zu

1 Vgl. Dennett, Daniel C.: »Quining Qualia«, in: Philosophy of mind. Classical and contemporary readings, Chalmers, David J. [Hrsg.], New York: Oxford University Press [zuerst 1988]/2002, S. 228-244.

2 Dennett nennt diesen »[...] my suggested fourfold essence of qualia [...]«. A.a.O., S. 229.

3 Churchland, Paul M.: »The Rediscovery of Light«, The Journal of Philosophy, [1996] Vol. 93, No. 5 , S. 223 . 
vernachlässigende angebliche $»$ Innerlichkeit«,$^{4}>$ Intrinsität $<,{ }^{5} »$ UnteilbarkeitsIntuition ${ }^{6},{ }^{2}$ Illusion der Homogenität,$^{7}{ }^{~}$ introspizierende Alltagspsychologie $\ll^{8}$ etc. beschränkt sind.

Die in dieser Arbeit geleistete Ausarbeitung eines phänomenal adäquaten Qualia-Begriffs hat gezeigt, dass wenn bspw. die auf der Nozizeption basierenden interozeptiven Empfindungen, wie Schmerz als Standardbeispiel für qualitatives resp. phänomenales Erleben gelten, dann müssen aber auch andere, bewusst erlebte somatoviszerale Empfindungen, wie propriozeptive und viszerale Rückmeldungsmuster, die einen zentralen Aspekt der Phänomenalität von Emotionen ausmachen, als phänomenale Qualitäten des Erlebens gelten (vgl. Abschnitt 8.2.3). Nicht nur körperlich bzw. sinnlich basierte Erlebniszustände der Art, wie-es-ist, Zahnschmerzen zu haben oder Glockengeläut zu hören, fallen unter den Qualia-Begriff, sondern auch Erlebniszustände der Art, wie-es-ist, ein ängstlich-beklemmendes Stechen in der Brust, im Hinblick auf eine persönliche, finanzielle Krise oder auf einen gewählten Präsidenten zu haben resp. eine tieftraurig-dumpfe Schwere im Rumpf, in Gedanken an den täglichen Hungertod von 18'ooo Kindern oder an eine schwere Enttäuschung durch einen nahestehenden Menschen zu empfinden. Deshalb ist der herkömmliche Qualia-Begriff insofern um Emotionen zu erweitern, als es für uns auch irgendwie ist, Emotionen bzw. eine mit ihnen unentwirrbar verschränkte Intentionalität somatoviszeraler Empfindungen ${ }^{9}$ zu erleben. ${ }^{10} \mathrm{Im}$

4 Metzinger, Thomas: Subjekt und Selbstmodell. Die Perspektivität phänomenalen Bewusstseins vor dem Hintergrund einer naturalistischen Theorie mentaler Repräsentation, Paderborn: Mentis 1999, S. 37. Vgl. dazu auch Dennett, Daniel C.: »Quining Qualia«, in: Philosophy of mind. Classical and contemporary readings, Chalmers, David J. [Hrsg.], New York: Oxford University Press [zuerst 1988]/2002, S. 229.

5 Dennett spricht diesbezüglich an mehreren Stellen von »intrinsic«. Vgl. z.B. A.a.O., S. 229, 241 und 244 .

6 Metzinger, Thomas: Subjekt und Selbstmodell. Die Perspektivität phänomenalen Bewusstseins vor dem Hintergrund einer naturalistischen Theorie mentaler Repräsentation, Paderborn: Mentis 1999, S. 36.

7 Genauer spricht Dennett von einer »doctrinal illusion of >homogeneity««. Dennett, Daniel C.: »Quining Qualia«, in: Philosophy of mind. Classical and contemporary readings, Chalmers, David J. [Hrsg.], New York: Oxford University Press [zuerst 1988]/2002, S. 243. Zur Konzeption von Qualia als vermeintlich »homogene« Entitäten vgl. auch Metzinger, Thomas: Subjekt und Selbstmodell. Die Perspektivität phänomenalen Bewusstseins vor dem Hintergrund einer naturalistischen Theorie mentaler Repräsentation, Paderborn: Mentis 1999, S. 36 .

8 A.a.O., S. 37.

9 Vgl. dazu z.B. Damasio, Antonio R.: Looking for Spinoza. Joy, sorrow and the feeling brain, London: Vintage 2004, S. 89.

10 Vgl. dazu auch Goldie, Peter: The emotions. A philosophical exploration, New York: Oxford University Press 2002, S. 4. 
Zusammenhang mit Goldies Konzeption des »feeling towards «11 und weil sich Formen von Intentionalität ausweisen lassen, die nicht von Phänomenalität getrennt werden können, ${ }^{12}$ umfasst eine phänomenal adäquate Konzeption von Qualia auch die damit verbundenen qualitativen Aspekte der Intentionalität von Emotionen bzw. der phänomenalen Intentionalität (vgl. Abschnitt 8.2.5). Zudem ist der Qualia-Begriff um die in Abschnitt 8.2.4 beschriebene Phänomenalität der für Emotionen grundlegenden Formen emotiv-kognitiver Erlebnisformen, im Sinne von Affektlogiken, ${ }^{13}$ »emotive thoughts « ${ }^{14}$ bzw. »emotional thoughts « ${ }^{15}$ und emotiven Modi des Denkens ${ }^{16}$ zu erweitern. Nicht zuletzt ist der Qualia-Begriff um zwei weitere Formen, in Abschnitt 8.2.6 beschriebenen nicht-emotiven, gedanklichen Formen des Erlebens zu erweitern: a.) um den Bereich phonologisch-verbaler bzw. generell auditiver, visueller, taktiler, olfaktorischer, gustatorischer und somatosensorischer ${ }^{17}$ Vorstellungserlebnisse, im Sinne herkömmlicher, aber imaginierter Sinnes-Qualia; ${ }^{18}$ b.) um eine weitere phänomenale Eigenschaft sui generis: ${ }^{19}$ Erlebniszustände des Verstehens und des Erfassens von Überzeugungen, die ganz allgemein darin bestehen, dass es irgendwie ist, sich als denkend, verstehend, urteilend zu erleben. ${ }^{20}$

Die in dieser Arbeit vorgenommene Revision des Qualia-Begriffs und der damit verbundene Nachweis der Ubiquität und Reichhaltigkeit von Qualia, zeigen auf, dass die Strategien, sie Zwecks leichterer Explizierbarkeit zu

11 A.a.O., S. 57.

12 Vgl. Graham, George / Horgan, Terence / Tienson, John: »Phenomenology, Intentionality, and the Unity of the Mind«, in: The Oxford Handbook of Philosophy of Mind, McLaughlin, Brian P. / Beckermann, Ansgar / Walter, Sven [Hrsg.], Oxford: Oxford University Press 2009, S. 53 o.

13 Vgl. Ciompi, Luc: Die emotionalen Grundlagen des Denkens. Entwurf einer fraktalen Affektlogik, Göttingen: Vandenhoeck \& Ruprecht 1997, S. 187-19o.

14 Pugmire, David: Rediscovering emotion, Edinburgh: Edinburgh University Press 1998, S. 63.

15 Stocker, Michael: »Emotional Thoughts«, American Philosophical Quarterly, [1987] Vol. 24, No. 1, S. 6 o.

16 Vgl. Damasio, Antonio R.: Looking for Spinoza. Joy, sorrow and the feeling brain, London: Vintage 2004, S. 84 .

17 Z.B. Schmerz bei der oben genannten Vorstellung eines Zahnarztbesuches.

18 Vgl. dazu auch Pitt, David: »The Phenomenology of Cognition or what is it Like to Think that P? «, Philosophy and Phenomenological Research, [2004] Vol. 69, No. 1, S. 3-4.

19 Vgl. dazu a.a.O., S. 4, 10 und 24.

20 Vgl. dazu Strawson, Galen: Mental reality, Cambridge, Massachusetts: MIT Press 1994, S. 8 und 12. Vgl. auch Soldati, Gianfranco: »Begriffliche Qualia. Zur Phänomenologie der Bedeutung«, in: Anatomie der Subjektivität. Bewusstsein, Selbstbewusstsein und Selbstgefühl, Grundmann, Thomas / Hofmann, Frank / Misselhorn, Catrin et al. [Hrsg.], Frankfurt am Main: Suhrkamp 2005, S. 145-147 und 149. 
transformieren oder auf einen besonders engen Begriff zu reduzieren und in der Folge zu >eliminieren<, aufgrund einer Verwirrung über den Untersuchungsgegenstand des hard problem scheitern müssen. Denn es ist nicht zulässig, Erleben auf transformierte ${ }^{21}>$ Qualia-Eigenschaften $<$ zu reduzieren oder aufgrund von irrtümlicherweise als notwendig22 vorausgesetzten Eigenschaften von Qualia zu behaupten, dass es keine Qualia bzw. keine phänomenalen Eigenschaften >gibt<. Nicht zuletzt ist es auch unzulässig, wenn man konstatiert, dass Qualia, weil sie angeblich nur »marginale $«^{23}$ oder seltsame Eigenschaften hätten, keine Relevanz für eine Theorie des Geistes hätten.

Wenn jemand z.B. behaupten würde, dass Licht-Quanten bzw. Photonen eine Ruhemasse aufweisen, und dann nachgewiesen wird, dass sie keine Ruhemasse haben, kann man dann die Schlussfolgerung ziehen, dass es kein Licht >gibt<?24 Oder stellen wir uns vor, jemand hätte vor 1927 behauptet, dass es nur dann Elektronen >gibt<, wenn sie zwingend und unter allen Umständen Partikeleigenschaften aufweisen. ${ }^{25}$ >Gibt< es nun keine Elektronen, wenn sich, entgegen einer solchen, zwar vereinfachten, aber zu engen und auch inadäquaten Definition herausgestellt hat, dass ihr Verhalten nicht darauf hinweist, dass sie nur Partikeleigenschaften, sondern paradoxerweise, je nach

21 So werden z.B. Farbqualia in nicht-phänomenale Eigenschaften intentionaler Repräsentationsgehalte oder in Oberflächeneigenschaften von Gegenständen bzw. in externe Eigenschaften oder Eigenschaften intentionaler Repräsentationsgehalte transformiert. Vgl. z.B. Lycan, William G.: Consciousness, Cambridge, Massachusetts: MIT Press 1987. Vgl. auch Tye, Michael: »Representationalist Theories of Consciousness«, in: The Oxford Handbook of Philosophy of Mind, McLaughlin, Brian P. / Beckermann, Ansgar / Walter, Sven [Hrsg.], Oxford: Oxford University Press 2009, S. 256 und 262. Vgl. ebenfalls Harman, Gilbert: »The intrinsic quality of experience «, in: Philosophy of mind. Critical concepts in philosophy. Volume 4: Consciousness, Crawford, Sean [Hrsg.], London: Routledge \& Kegan Paul [zuerst 199o]/2011, S. 110.

22 Wie z.B. die angeblichen Eigenschaften der Infallibilität, Unmittelbarkeit, NichtIntentionalität, Introspektivität, Homogenität und Atomizität. Vgl. Abschnitt 9.2.

23 Churchland, Paul M.: »The Rediscovery of Light«, The Journal of Philosophy, [1996] Vol. 93, No. 5 , S. 223 .

24 Mehr darüber, dass Photonen keine Ruhemasse haben, vgl. Dorn, Friedrich / [Hrsg.: Oberholz, Heinz-Werner]: Dorn-Bader Physik in einem Band, Braunschweig: Schroedel 2013, S. 546 .

25 De Broglie stellte 1924 die These auf, dass auch klassische Materieteilchen wie Elektronen nicht nur Teilchencharakter, sondern auch einen Wellencharakter aufweisen müssen, was 1927 (Beugung von einem Elektronenstrahl an einem Nickel-Kristall von Lester Germer und Clinton Davisson) und 1961 (Doppelspaltexperiment von Claus Jönsson) experimentell bestätigt wurde. Vgl. dazu a.a.O., S. 510-511. Vgl. dazu auch Mainzer, Klaus: »Korpuskel-Welle-Dualismus«, in: Enzyklopädie Philosophie und Wissenschaftstheorie. Band 2: $H$-o, Mittelstrass, Jürgen [Hrsg.], Stuttgart: Metzler 2004, S. 477-478. 
Versuchsanordnung, auch Welleneigenschaften aufweisen? ${ }^{26}$ Freilich kann man nun behaupten, dass es keine Elektronen 'gibt<, die sich immer so verhalten, wie sich Partikel verhalten würden. Freilich wäre es richtig, wenn jemand sagen würde, dass es keine Elektronen >gibt ‘, die jener einfachen, engen und veralteten Definition unseres Gedankenexperimentes entsprechen. Aber dann ist nur nachgewiesen, dass eine bestimmte Konzeption über Elektronen nicht richtig ist, nicht aber, dass es keine Elektronen >gibt<.

So kann bspw. Dennett, wenn er aufzeigt, dass unsere Urteile über (vergangene) Qualia nicht infallibel sind und dass kognitive Prozesse und phänomenales Erleben schwer zu trennen sind, mit Recht behaupten, dass Qualia in dieser Hinsicht - oder vielleicht auch grundsätzlich - fallibel sind. Wenn wir ihm zugestehen, dass er mit seinen >Intuitionspumpen< dies nachgewiesen hat, dann hat auch er nur gezeigt, dass eine bestimmte Konzeption über phänomenales Erleben falsch ist. Leider hat er damit aber bloss aufgezeigt, dass es keine Qualia gibt, die dieser (und offenbar auch seiner) Konzeption entsprechen. Der intuitive Sprung von der Feststellung, dass es keine Qualia gibt, die (s)einem »vierfältigen ${ }^{27}$ Konstrukt entsprechen, zur ontologischen Behauptung, dass es grundsätzlich kein phänomenales Erleben und keine

26 Dies ist insofern paradox, als dass Teilchen- bzw. Wellennatur von Elektronen nach klassischem Verständnis inkompatible Eigenschaften sind, da sie »[...] verschiedenen, wechselseitig inkompatiblen Versuchsanordnungen [...] « entsprechen, die nicht gleichzeitig durchgeführt werden können. Polkinghorne, John C.: Quantentheorie. Eine Einführung, Stuttgart: Philipp Reclam Jun 2011, S. 66. Denn entweder man nähert sich dem Elektron mit einem Doppelspaltexperiment, indem man es »[...] mit Fragen über seine Wellennatur untersucht $[\ldots]$ « und dann eine »wellenmässige « Antwort« in Form eines Interferenzmusters erhält; oder man untersucht es mit einer Versuchsanordnung, die für ein Teilchenexperiment eingerichtet ist, indem man beobachtet, durch welchen der beiden Spalten sich das Elektron bewegt. Beobachtet man das Elektron mit letzterer Versuchsanordnung, so erhält man eine »teilchenmässige « Antwort«, die den gemessenen Auftreffpunkten der Elektronen hinter den Spalten entspricht. Ebd. Wenn aber beide alternativen, sich ausschliessenden Eigenschaften von Elektronen durch Experimente bestätigt wurden (z.B. durch das oben erwähnte Doppelspaltexperiment von Claus Jönsson), so widerspricht dies dem Aristotelischen Gesetz vom ausgeschlossenen Dritten: es dürfte eigentlich keine dritte Möglichkeit zwischen `Teilchen« und `Nicht-Teilchenく geben, was heisst, dass etwas entweder ein Teilchen ist oder eben keinTeilchen ist. Niels Bohr reagierte auf diese Widersprüchlichkeit, indem er den Begriff der >Komplementarität< einführte: ein Elektron ist nicht entweder als Teilchen oder als Welle zu verstehen, sondern hat sowohl Teilchen- als auch Wellennatur auch wenn beide Eigenschaften unserer Alltagsintuition als >unvereinbar< erscheinen. Vgl. dazu auch a.a.O., S. 44-49, 65 und 68 .

27 Dennett, Daniel C.: »Quining Qualia «, in: Philosophy of mind. Classical and contemporary readings, Chalmers, David J. [Hrsg.], New York: Oxford University Press [zuerst 1988]/2002, S. 229 . 
Qualia >gibt‘, ist deshalb nicht gerechtfertigt - und basiert gerade aus diesem Grund, wie oben bemerkt, nur auf den von ihm flüchtig in Erwägung gezogenen Strohmann-Argumenten. ${ }^{28}$ Da er sich jedoch keinen anderen QualiaBegriff vorstellen kann, ${ }^{29}$ ist für ihn die Sache relativ schnell erledigt: "So when we look one last time at our original characterization of qualia [...] we find that there is nothing to fill the bill ${ }^{30}$

Mit der in dieser Arbeit vorgenommenen Ausarbeitung eines phänomenal adäquaten Qualia-Begriffs und der damit einhergehenden Kritik an herkömmlichen, verengten und viel zu einfachen Qualia-Begriffen lässt sich deshalb nicht zuletzt aufzeigen, dass eliminativistische, identitätstheoretische und reduktiv-funktionalistische bzw. reduktiv-repräsentationalistische Theorien des Geistes die den Untersuchungsgegenstand betreffende und notwendige Vorarbeit ihrer Theorien vernachlässigt haben. ${ }^{31}$ Diese hätte darin bestanden, zunächst das Explanandum >Qualia $<$ bzw. >bewusstes Erleben $<$ hinreichend zu bestimmen.

\subsection{Falsifizierung angeblich »essenzieller« Qualia-Eigenschaften}

Meine Kritik von evasiven oder verengten, schlicht: phänomenal inadäquaten Qualia-Konzeptionen soll nun aufzeigen, dass ein Qualia-Begriff, der nur einen Bruchteil phänomenalen Erlebens abdeckt oder der Phänomenalität Eigenschaften unterstellt, die für bewusstes Erleben weder notwendig noch hinreichend sind, keine nützliche Basis sein kann, um das hard problem anzugehen. Umso mehr erweist sich ein phänomenal inadäquater Qualia-Begriff als unbrauchbare Grundlage, um Phänomenales zu eliminieren oder um zu

28 Vgl. dazu auch ebd.

29 Dennett gesteht paradoxerweise ein, dass wir keine Zombies sind: »[...] I don't deny the reality of conscious experience [...] I grant that conscious experience has properties«. A.a.O., S. 227. Im Hinblick auf die von ihm postulierten, seltsamen Eigenschaften von bewusstem Erleben nimmt er jedoch wieder implizit wieder Zuflucht auf seinen restriktiven Qualia-Begriff: »[...] but these properties are so unlike the properties traditionally imputed to consciousness [...]. My claim [...] is that conscious experience has no properties that are special in any of the ways qualia have been supposed to be special«. Ebd. [Hervorhebung im Original]. Doch selbst wenn bewusstes Erleben nicht jene »speziellen« Eigenschaften hat, ist damit weder Phänomenalität noch das hard problem >eliminiert<. Mit »speziell« meint Dennett wohl wieder die Eigenschaften seines vierfältigen Qualia-Begriffs (nicht-begrifflich, intrinsisch, privat, unmittelbar). Vgl. a.a.O., S. 229.

3o A.a.O., S. 244 [Hervorhebung E.E.].

31 Das heisst nicht, dass reduktionskritische Ansätze >antiphysikalistisch $<$ bzw. substanzdualistisch sein müssen (vgl. weiter unten, Abschnitt 10.3). 
begründen, warum Qualia auf Dritte-Person-Eigenschaften reduzierbar sein sollen. Denn mit welchem Recht sollen sich, auf der Grundlage von angeblichen Qualia-Eigenschaften, die nicht zwingend oder sogar überhaupt keine Eigenschaften von phänomenalem Erleben sind, Qualia in toto leugnen lassen?

So sind zum Beispiel die nach Dennett angeblich »essenziellen « ${ }^{32}$ Eigenschaften der $>$ Unmittelbarkeit ${ }^{33}$ bzw. der $>$ Infallibilität< von Urteilen $\ddot{u} b e r$ (vergangene) Erlebnisse weder notwendige noch hinreichende Voraussetzungen für phänomenales Erleben. Denn warum soll der Nachweis von Dennetts Kaffeetestern ${ }^{34}$, dass ein vergangenes phänomenales Erlebnis heute in einem anderen Licht erscheinen kann, ${ }^{35}$ belegen, dass das Explanandum des hard problem bei »näherem Hinsehen $\aleph^{36}$ vielleicht gar »nicht existiert«? ${ }^{37}$ Mit einem phänomenal adäquaten Qualia-Begriff können wir mit Ciompi, ${ }^{38}$ Engelen, ${ }^{39}$ Pugmire, ${ }^{40}$ Stocker, ${ }^{41}$ Chalmers ${ }^{42}$ gerade aufzeigen, dass sich das

32 A.a.O., S. 228.

33 Vgl. z.B. McDonald, Cynthia: »Introspection«, in: The Oxford Handbook of Philosophy of Mind, McLaughlin, Brian P. / Beckermann, Ansgar / Walter, Sven [Hrsg.], Oxford: Oxford University Press 2009, S. 741. Oder vgl. Dennett, der diesbezüglich von »[...] directly apprehensible properties of experience [...]« spricht. Dennett, Daniel C.: »Quining Qualia«, in: Philosophy of mind. Classical and contemporary readings, Chalmers, David J. [Hrsg.], New York: Oxford University Press [zuerst 1988]/2002, S. 229.

34 Vgl. a.a.O., S. 231-234.

35 Freilich ist das Gedankenexperiment von Dennetts Kaffeetestern Chase und Sanborn etwas komplizierter aufgebaut. Dennett will damit auch nachweisen, dass es für Chase und Sanborn schwierig ist, bei einer Veränderung des Geschmackerlebens zu bestimmen, ob sich nun das phänomenale Erleben des Geschmackes oder ihr Urteil über bzw. ihre Reaktion auf den Geschmack oder beides geändert hat. A.a.O., S. 232-233. Dies zeigt jedoch, wie ich in Abschnitt 8.1 aufgezeigt habe, nur auf, dass Urteile über (vergangene) Qualia fallibel sind und dass kognitive Prozesse und phänomenales Erleben interdependente Phänomene sind.

36 Metzinger, Thomas [Hrsg.]: Grundkurs Philosophie des Geistes. Band 1: Phänomenales Bewusstsein, Paderborn: Mentis 2007, S. 204.

37 Ebd. Dies zieht auch Metzinger in Erwägung, weil wir phänomenale Begriffe von einem Auftreten zum nächsten nicht zuverlässig reidentifizieren können.

38 Vgl. Ciompi, Luc: Die emotionalen Grundlagen des Denkens. Entwurf einer fraktalen Affektlogik, Göttingen: Vandenhoeck \& Ruprecht 1997, S. 183-192.

39 Vgl. Engelen, Eva-Maria: Gefühle, Stuttgart: Reclam 2007, S. 33.

40 Zum Zusammenhang zwischen propositionalen Gehalten, somatischen Empfindungen, Gedanken und Gefühlen vgl. auch Pugmire, David: Rediscovering emotion, Edinburgh: Edinburgh University Press 1998, S. 94-105.

$41 \mathrm{Zu}$ >wutgefärbten` Gedanken bzw. zum Fühlen, Denken und Handeln aus (und aufgrund von) Wut vgl. auch Stocker, Michael: »Einige Betrachtungen zu intellektuellen Wünschen und Emotionen «, in: Philosophie der Gefühle, Döring, Sabine [Hrsg.], Frankfurt am Main: Suhrkamp [zuerst 2004, engl.]/2009, S. 214.

42 Vgl. Chalmers, David J.: The conscious mind. In search of a fundamental theory, New York: Oxford University Press 1996, S. 10. 
gesamte Feld unseres Erlebens bspw. je nach unserer Grundstimmung »färbt « ${ }^{43}$ und dass Emotionen unser gesamtes phänomenales Erleben auf ihre je eigene charakteristische Weise durchdringen, prägen und verändern. ${ }^{44}$ Das bedeutet, wie ich noch zeigen werde, dass phänomenales Erleben keineswegs aus angeblich »essenziellen $\ll, 45$ »einfachen $\ll,{ }^{46}>$ unmittelbaren ${ }^{47}$ oder $>$ unveränderlichen Qualia (mit einer wieder erkennbaren »Selbigkeit « ${ }^{48}$ ) bestehen muss. Wenn sich dieser statisch-atomistische Qualia-Begriff von Dennett ${ }^{49}$ oder Metzinger $^{50}$ als falsch erweist, wie soll er dann, auch nur im Ansatz aufzeigen, dass phänomenales Erleben kein Explanandum darstellt? Zeigt die Falsifizierung dieses vereinfachten und verengten Qualia-Begriffs nicht sogar vielmehr auf, dass es sich bei phänomenalem Erleben um ein für das hard problem noch schwierigeres Explanandum handelt, als allgemein angenommen wird?

43 Ciompi, Luc: Die emotionalen Grundlagen des Denkens. Entwurf einer fraktalen Affektlogik, Göttingen: Vandenhoeck \& Ruprecht 1997, S. 96.

44 Zur Metapher des >Färbens (»color «) unseres phänomenalen Erlebens durch Emotionen vgl. auch Chalmers, David J.: The conscious mind. In search of a fundamental theory, New York: Oxford University Press 1996, S. 10.

45 Dennett, Daniel C.: »Quining Qualia«, in: Philosophy of mind. Classical and contemporary readings, Chalmers, David J. [Hrsg.], New York: Oxford University Press [zuerst 1988]/2002, S. 228.

46 So z.B. Metzingers Qualia-Konzeption, vgl. Metzinger, Thomas [Hrsg.]: Grundkurs Philosophie des Geistes. Band 1: Phänomenales Bewusstsein, Paderborn: Mentis 2007, S. 203.

47 So bspw. Dennett, für den Qualia »directly apprehensible« sind. Vgl. Dennett, Daniel C.: »Quining Qualia«, in: Philosophy of mind. Classical and contemporary readings, Chalmers, David J. [Hrsg.], New York: Oxford University Press [zuerst 1988]/2002, S. 244.

48 Metzinger, Thomas [Hrsg.]: Grundkurs Philosophie des Geistes. Band 1: Phänomenales Bewusstsein, Paderborn: Mentis 2007, S. 203.

49 Vgl. Dennett, Daniel C.: »Quining Qualia«, in: Philosophy of mind. Classical and contemporary readings, Chalmers, David J. [Hrsg.], New York: Oxford University Press [zuerst 1988]/2002, S. 228.

$50 \quad$ Vgl. dazu Metzinger, Thomas: Subjekt und Selbstmodell. Die Perspektivität phänomenalen Bewusstseins vor dem Hintergrund einer naturalistischen Theorie mentaler Repräsentation, Paderborn: Mentis 1999, S. 33 und 36. Vgl. ebenfalls Metzinger, Thomas [Hrsg.]: Grundkurs Philosophie des Geistes. Band 1: Phänomenales Bewusstsein, Paderborn: Mentis 2007, S. 58. Metzinger führt jedoch selber auch Gegenargumente zu diesem atomistischen QualiaBegriff auf: Vgl. dazu Metzinger, Thomas: Subjekt und Selbstmodell. Die Perspektivität phänomenalen Bewusstseins vor dem Hintergrund einer naturalistischen Theorie mentaler Repräsentation, Paderborn: Mentis 1999, S. 36. Zur monadischen Konzeption von Qualia vgl. auch Lycan, William: Representational Theories of Consciousness «, in: The Stanford Encyclopedia of Philosophy, 2008, Online: http://plato.stanford.edu/archives/fall2008/ entries/consciousness-representational/ [zuletzt geprüft am 28.o9.2018], Abschnitt 1.o. 
Oder wie soll bspw. die These von Tye, Qualia bestünden in (nichtbegrifflichen) repräsentationalen intentionalen Gehalten, ${ }^{51}$ begründen, dass Qualia auf diese Eigenschaften reduzierbar und durch sie erklärt werden können sollen, wenn Intentionalität selbst phänomenale Eigenschaften aufweist? Und warum sollen externalistisch-repräsentationalistische ${ }^{52}$ und teleosemantische ${ }^{53}$ Theorien des Geistes das hard problem lösen, bzw. Qualia auf die angeblich leichtere Naturalisierbarkeit ${ }^{54}$ von intentionalen Gehalten reduziert werden können, wenn sich Formen von Intentionalität ausweisen lassen, die nicht von Phänomenalität getrennt werden können? ${ }^{55}$

Im Rahmen dieser Arbeit ist es nicht möglich, die Konsequenzen aller vorgefundenen, phänomenal inadäquaten Behauptungen über vermeintliche Qualia-Eigenschaften aufzuzeigen. In diesem Kapitel werde ich deshalb vor allem auf einige prominente Beispiele, angeblich »essenzieller « ${ }^{56}$ Eigenschaften phänomenalen Erlebens (wie beispielsweise auf Eigenschaften in Dennetts Konzeption seines »vierfältigen « ${ }^{57}$ Qualia-Begriffs) eingehen.

\subsection{1 >Infallibilität< und >Unmittelbarkeit<}

>Infallibilität< ist, wenn sich diese auf eine Wahrheit im propositionalen Sinne beziehen soll, ${ }^{58}$ nicht notwendigerweise eine Eigenschaft von Qualia. Ein

$5^{1} \quad$ Vgl. z.B. Tye, Michael: »Representationalist Theories of Consciousness«, in: The Oxford Handbook of Philosophy of Mind, McLaughlin, Brian P. / Beckermann, Ansgar / Walter, Sven [Hrsg.], Oxford: Oxford University Press 2009, S. 262.

52 Vgl. dazu ebd.

53 Vgl. dazu Dretske, Fred:Naturalizing the mind, Cambridge, Massachusetts: MIT Press 1995. Vgl. auch Dennett, Daniel C.: »Evolution, Error and Intentionality«, in: The foundations of artificial intelligence. A sourcebook, Partridge, Derek / Wilks, Yorick [Hrsg.], Cambridge, New York: Cambridge University Press 2010, S. 190-211.

54 Vgl. Lycan, William: »Representational Theories of Consciousness«, in: The Stanford Encyclopedia of Philosophy, 2008, Online: http://plato.stanford.edu/archives/fall20o8/ entries/consciousness-representational/ [zuletzt geprüft am 28.o9.2018], Abschnitt 2.4. Vgl. z.B. Graham, George / Horgan, Terence / Tienson, John: »Phenomenology, Intentionality, and the Unity of the Mind«, in: The Oxford Handbook of Philosophy of Mind, McLaughlin, Brian P. / Beckermann, Ansgar / Walter, Sven [Hrsg.], Oxford: Oxford University Press 2009, S. 530. Vgl. ebenfalls Goldie, Peter: The emotions. A philosophical exploration, New York: Oxford University Press 2002, S. 4, 54-55.

56 Dennett, Daniel C.: »Quining Qualia «, in: Philosophy of mind. Classical and contemporary readings, Chalmers, David J. [Hrsg.], New York: Oxford University Press [zuerst 1988]/2002, S. 228.

57 A.a.O., S. 229. (Wie oben erwähnt definiert Dennett Qualia als unbeschreiblich, intrinsisch, privat und unmittelbar.)

$5^{8}$ Im Hinblick auf die angebliche Qualia-Eigenschaft der >Infallibilität ist es wichtig, zu unterscheiden zwischen einer nicht vorhandenen >Infallibilitätく von Urteilen über phänomenales Erleben und der bei phänomenalem Erleben vorhandenen >Infallibilität‘, 
korrektes Urteil über das, was ich phänomenal erlebe, stellt keine Bedingung dar, um dieses Erlebnis haben zu können: Selbst wenn ich, aufgrund einer optischen Täuschung, das falsche Urteil fälle, dass die rotierende, schwarzweisse Benham-Scheibe vor mir >farbig « sei, so heisst das doch nicht, dass ich kein Farberleben in Form einer optischen Täuschung habe. ${ }^{59}$

Auch im etwas komplizierteren Fall des Gedankenexperiments von Dennett mit den Kaffeetestern Chase und Sanborn ${ }^{60}$ verhält es sich grundsätzlich nicht anders: Wenn ich mich nicht mehr genau erinnere, wie es war, den Kaffeegeschmack von gestern zu erleben, und ich nicht weiss, ob sich meine Reaktion auf den Geschmack oder der Geschmack selbst verändert hat, so löst sich damit doch nicht rückwirkend das gestrige Erleben des Geschmacks auf - und doch auch nicht mein, aufgrund meiner jetzigen Reaktion auf diesen Geschmack, modifiziertes Erleben. ${ }^{61}$ Heute mag meine Erinnerung an das

insofern, dass phänomenale Bewusstseinszustände eine Wissensform mit pour-soiStruktur darstellen, die nicht auf Wahrheit in einem propositionalen Sinne bezogen ist. Vgl. dazu auch Schildknecht, Christiane: Sense and self. Perspectives on nonpropositionality, Paderborn: Mentis 2002, S. 224-226. Vgl. ebenfalls Schildknecht, Christiane: Aspekte des Nichtpropositionalen, Bonn: Bouvier 1999, S. 11. Wenn wir eine Schokolade anschauen, so kann das damit verbundene Erleben ihrer >braunen< Farbe insofern als >infallibel< gelten, dass es auf eine ganz bestimmte Weise, d.h., irgendwie ist, die Schokolade fälschlicherweise als etwas >Braunes< zu sehen. Eine Schokolade müssten wir aber >eigentlichく als etwas `Orangefarbenes` erleben (weil Braun gar keine Spektralfarbe von Licht ist und die für Brauntöne typischen Wellenlängen solche sind, die von orangefarbenen und gelben Oberflächen reflektiert werden). Dennoch ist das nicht-propositionale Wissen, wie es ist, diese optische Täuschung zu erleben, nicht von der Richtigkeit des Urteils über die >wirklicheく Farbe der Schokolade abhängig. Insofern können Qualia also als >infallibel< und >unmittelbar< betrachtet werden, denn es ist unsinnig, dem >Braunerleben< als solchem, zu unterstellen, dass es nur ein >vermeintliches Braunerleben` sei: die Farbe >Braun « wird erlebt. Im Hinblick auf dieses >Braunerleben $<$ spielt es keine Rolle, dass die Oberflächeneigenschaften der Schokolade eher einem Orange als einem Braun entsprechen. Vgl. dazu auch Lanz, Peter: Das phänomenale Bewusstsein. Eine Verteidigung, Frankfurt am Main: Klostermann 1996, S. 154-155.

59 Wie oben erwähnt, wird die schwarz-weisse Benham-Scheibe, wenn sie rotiert, aufgrund einer optischen Täuschung als etwas >Farbiges` wahrgenommen. Vgl. dazu Staudacher, Alexander: Phänomenales Bewusstsein als Problem für den Materialismus, Berlin: De Gruyter 2002, S. 324.

6o Vgl. dazu auch Dennett, Daniel C.: »Quining Qualia«, in: Philosophy of mind. Classical and contemporary readings, Chalmers, David J. [Hrsg.], New York: Oxford University Press [zuerst 1988]/2002, S. 231-234.

61 Der Infallibilitäts-Begriff, so wie ihn Dennett verwendet, zielt also auf diachrone oder andere epistemische Fragen der Verifizierung ab, und nicht darauf, dass das Bier im Moment gerade so und so schmeckt. Eine `Täuschungく im Hinblick auf das Geschmacksempfinden von Gestern ist epistemisch relevant, nicht aber phänomenal relevant. Das Bier schmeckt eben so, wie es im Moment schmeckt, selbst wenn es (bspw. aufgrund einer 
Erleben von gestern verblasst sein oder anders erscheinen als es war, das zeigt aber in keiner Weise auf, dass ich gestern >eigentlich < ein Zombie war und in »Wirklichkeit« ${ }^{62}$ keine Qualia hatte bzw. >nichts< erlebte. ${ }^{63}$

Ergo: selbst wenn ich nicht weiss, ob sich nur das Erleben oder vielleicht auch (oder nur) meine Reaktion auf den Geschmack geändert hat, so zeigt dies nur auf, dass (wie in den Abschnitten 8.2.3 bis 8.2.5 aufgezeigt) kognitive Prozesse und phänomenales Erleben schwer zu trennen sind, weil sie interdependente Phänomene sind. In dieser Hinsicht wären Qualia im herkömmlichen Sinne, wenn man sie fälschlicherweise als »einfachen «64 Inhalt isolieren wollte, d.h. unabhängig von kognitiven Prozessen zu betrachten versuchte, auch nicht > unmittelbar<. So werden beispielsweise beim Erleben einer Emotion somatoviszerale Zustände nicht >unmittelbar<, d.h. unabhängig von kognitiven Prozessen erlebt. Denn letztere verleihen dem somatischen Erleben eine emotionale Begrifflichkeit ${ }^{65}$ und eine Intentionalität. ${ }^{66}$ Die Phänomenalität bei emotionalem Erleben besteht gerade darin, dass mit ihm kognitives und somatisches Erleben interagieren können und sich dies als >emotiv-kognitivsomatisches Erleben< zeigt. ${ }^{67}$

Hypnose) wie Cola schmecken würde, wäre dies nur in epistemischer Hinsicht relevant in phänomenaler Hinsicht ist es sogar irrelevant, ob Bier, aufgrund einer Hypnose, nach Cola schmeckt. Selbst der, wegen einer Hypnose erlebte Cola-Geschmack, kann insofern als eine >infallible< phänomenale Tatsache gelten, dass das Bier, wegen der Hypnose, in diesem Moment des Schmeckens eben genau wie Cola (und nicht anders) erlebt wird. Vgl. dazu auch Searle, John R.: The mystery of consciousness, New York: New York Review Books 1997, S. 112.

62 So stellt Metzinger, in einer Einleitung zu Dennetts Aufsatz Quining Qualia die Frage: »Existiert in Wirklichkeit überhaupt so etwas wie >das Quale selbst< [...]?«. Metzinger, Thomas [Hrsg.]: Grundkurs Philosophie des Geistes. Band 1: Phänomenales Bewusstsein, Paderborn: Mentis 2007, S. 203.

63 Vgl. dazu auch Dennett, Daniel C.: »Quining Qualia«, in: Philosophy of mind. Classical and contemporary readings, Chalmers, David J. [Hrsg.], New York: Oxford University Press [zuerst 1988]/2002, S. 232-233.

64 So z.B. Metzingers Qualia-Konzeption, vgl. Metzinger, Thomas [Hrsg.]: Grundkurs Philosophie des Geistes. Band 1: Phänomenales Bewusstsein, Paderborn: Mentis 2007, S. 203.

65 Vgl. dazu Engelen, Eva-Maria: Gefühle, Stuttgart: Reclam 2007, S. 33.

66 Vgl. Z.B. Goldie, Peter: The emotions. A philosophical exploration, New York: Oxford University Press 2002, S. 4. Vgl. dazu auch Damasio, Antonio R.: Looking for Spinoza. Joy, sorrow and the feeling brain, London: Vintage 2004, S. 89.

67 Analog zum Begriff der >Infallibilität, gilt es auch hier, fest zu halten, dass der Art und Weise, wie es ist, das Zusammenspiel von kognitiven Prozessen und somatoviszeralen Empfindungen als Gefühl zu erleben (als Ganzes) eine >Unmittelbarkeit`zugesprochen werden könnte. Selbst wenn ich mich bspw. durch den kognitiven Prozess des Schönredens über meine ursprüngliche Emotion täusche, so hat das Erleben dieser 
Auch im Hinblick auf den herkömmlichen Qualia-Begriff sinnlichphänomenalen Erlebens kann nicht davon ausgegangen werden, dass Sinneserlebnisse >unmittelbar und isoliert von kognitiv-emotiven Faktoren ablaufen: So weist derWissenschaftstheoretiker Alan F. Chalmers darauf hin, dass unsere Wahrnehmung nie unmittelbar ist. ${ }^{68}$ Das, was wir phänomenal sehen, ist nicht direkt durch die Retina bzw. die Netzhaut auf der Innenseite des Auges bestimmt, sondern hängt auch wesentlich von unserer Kultur und von kognitiven Fähigkeiten bzw. Faktoren ab. So haben bspw. bei identischen Gegebenheiten, $»[\ldots]$ erfahrene und geschulte Beobachter nicht die gleichen Wahrnehmungsfähigkeiten wie der Novize «.69 Selbstverständlich ist die Repräsentation auf der Netzhaut eine Teilursache, für das was wir sehen. Ein sehr wichtiger Teil unserer Wahrnehmung wird jedoch nicht »[...] von den physikalischen Eigenschaften unserer Augen $[\ldots] \ll^{70}$ bestimmt, sondern auch von kognitivemotiven Faktoren, wie kulturbedingter Erziehung, unseren Erwartungen, vom »inneren Zustand unseres Gemüts « ${ }^{71}$ und nicht zuletzt der Prägung unseres Verstandes. ${ }^{72}$ So haben beispielsweise Experimente gezeigt, dass Subjekte von Kulturen, bei welchen es nicht üblich ist, dreidimensionale Gegenstände zweidimensional darzustellen, einfache perspektivische Zeichnungen, wie z.B. die von einer Treppe, nicht als dreidimensionale Darstellung einer Treppe sehen, sondern nur als ein Linienmuster. ${ }^{73}$ Das bedeutet: unsere Vorstellungen und Erwartungen durchdringen unseren Wahrnehmungsraum. Wahrnehmungsmuster können folglich als eine mit Wahrnehmung simultan auftretende Vorstellung aufgefasst werden, die konstitutiv für das Muster einer Wahrnehmung ist: »Compare forming an image of a duck and seeing lines on paper as a duck: the duck image seems to have made a trip from imaginative space to real perceived space « ${ }^{74}$ Das bedeutet, dass selbst einfache Fallbeispiele visueller Qualia von Linienmustern ein Beleg für die Interdependenz von kognitiven

manipulierten Emotionalität ihre >Unmittelbarkeit<, denn es ist auch irgendwie diese Selbstmanipulation zu erleben.

68 Vgl. Chalmers, Alan F. / [Hrsg.: Bergemann Niels / Altstötter-Gleich Christine]: Wege der Wissenschaft. Einführung in die Wissenschaftstheorie, Berlin: Springer [zuerst 1976, engl.]/2001, S. 8-12.

69 A.a.O., S. 11.

70 Ebd.

71 A.a.O., S. 12.

72 Vgl. a.a.O., S. 11-12.

73 Vgl. a.a.O., S. 9.

74 McGinn, Colin: Mindsight. Image, dream, meaning, Cambridge, Massachusetts: Harvard University Press 2006, S. 5 o. 
und sinnlichen Fähigkeiten bzw. Erlebnisqualitäten sind. ${ }^{75}$ Ebenfalls zeigen die in Abschnitt 8.2.4 dargelegten Fälle emotiv-kognitiven Erlebens auf, dass unsere sinnliche Wahrnehmung vom Gemütszustand mitgeformt wird. ${ }^{76}$ Selbst wenn es also schwierig sein sollte, die von Dennett aufgeworfenen Frage über svermeintliche < oder `wirkliche< Veränderungen des Geschmackerlebens, zu beantworten (bzw. ob sich nun das phänomenale Erleben des Geschmackes oder unser Urteil über den Geschmack oder beides geändert hat), ist damit nur gezeigt, dass (eine so definierte) >Infallibilität $<$ und >Unmittelbarkeit nicht notwendigerweise eine Eigenschaft von Qualia ist.

Zudem macht die Unterscheidung zwischen einer >vermeintlichen< oder >wirklichen< Veränderung des Geschmackerlebens ${ }^{77}$ - wenn es um subjektives Erleben geht (!) - keinen Sinn, denn phänomenal betrachtet ist in solch einer Situation das >vermeintliche< gleichbedeutend mit dem >wirklichen< Erleben. ${ }^{78}$

But couldn't we disprove the existence of these data [bewusstes Erleben bzw. Phänomenalität] by proving that they are only illusions? No you can't disprove the existence of conscious experiences by proving that they are only an appearance [...] because where consciousness is concerned the existence of the appearance is the reality. [...] This is not an epistemic point. ${ }^{79}$

Eliminativisten und Qualia-Skeptiker stützen sich dennoch nicht selten auf solche spezifische Formen der Fallibilität von Urteilen über vergangenes phänomenales Erleben oder über die Fallibilität der Beurteilung eigener

75 Dass die kognitive Fähigkeit, Vorstellungen zu formen, ebenfalls Erlebnisqualitäten aufweist, habe ich in Abschnitt 8.2.6 aufgezeigt.

76 Vgl. Chalmers, Alan F. / [Hrsg.: Bergemann Niels / Altstötter-Gleich Christine]: Wege der Wissenschaft. Einführung in die Wissenschaftstheorie, Berlin: Springer [zuerst 1976, engl.]/2001, S. 12 .

77 Vgl. dazu auch Dennett, Daniel C.: »Quining Qualia «, in: Philosophy of mind. Classical and contemporary readings, Chalmers, David J. [Hrsg.], New York: Oxford University Press [zuerst 1988]/2002, S. 232-233.

78 In diesem Zusammenhang stellt Seager fest, dass gerade die Konzeption, dass das hard problem vielleicht nur eine »kognitive Illusion« (»cognitive illusion«) sei, wieder auf phänomenales Bewusstsein zurückverweist: »[...] the illusion requires that conscious states can have two $>$ modes of presentation $<$ (as qualitative consciousness to introspection, as a brain state to empirical investigation) [...] and this tends to lead right back to their being special non-physical properties by which we are aware of our experience«. Seager, William: »Emergentist Panpsychism«, Journal of Consciousness Studies, [2012] Vol. 19, No. 9-10, S. 29 [Hervorhebung E. E.].

79 Searle, John R.: The mystery of consciousness, New York: New York Review Books 1997, S. 112 [Hervorhebung im Original, Ergänzung in Klammern von E. E.]. 
Wahrnehmungsfähigkeiten ${ }^{80}$ oder des auf den Körper bezogenen IchGefühles ${ }^{81}$ etc. Der Grund dafür scheint (analog zur fallibilistischen Kritik der Analytischen Philosophie in der Mitte des 20. Jh. an der Sinnesdatentheorie) ${ }^{82}$ in der Annahme zu liegen, dass Qualia »erkenntnistheoretisch verzichtbar ${ }^{83}$

8o Als weiteres Beispiel seien hier noch Fehleinschätzungen der eigenen Wahrnehmungsfähigkeit (die auch Fehleinschätzungen der Bewegungsfähigkeiten einschliessen können) aufgeführt, die ihre Ursache in neurologischen Schädigungen bestimmter Hirnregionen haben: es handelt sich dabei um die ganz besonders seltsamen Fälle von Anosognosie bzw. anosognosia. Patienten mit dieser Bewusstseinsstörung leugnen ihre Wahrnehmungsfähigkeits- oder Bewegungsfähigkeits-Defizite, auch wenn diese noch so massiv sind. Vgl. dazu Damasio, Antonio R.: The feeling of what happens. Body, emotion and the making of consciousness, London: Vintage 2000, S. 209-213. So leugnen Patienten mit dem Anton's Syndrom (eine spezifische Form von Anosognosie) sogar, dass sie blind sind: »[...] subjects are blind but think and report that they see«. Block, Ned J.: »Consciousness and Cognitive Access«, Proceedings of the Aristotelian Society, [2008] Vol. 108, No. 1 (Part 3: October), S. 296. Weitere klassische Beispiele für Anosognosie sind Damasio zufolge Patienten mit einseitigen Körperlähmungen (Hemiplegie), die behaupten, dass sie keinerlei Lähmungserscheinungen hätten. Damasio, Antonio R.: The feeling of what happens. Body, emotion and the making of consciousness, London: Vintage 2000, S. 210-211.

Nach Block sind solche Fehleinschätzungen der eigenen Wahrnehmungsfähigkeit zu einem gewissen Grad bei normalen Subjekten, bspw. unter Stress, auch nachweisbar. Vgl. Block, Ned J.: »Consciousness and Cognitive Access «, Proceedings of the Aristotelian Society, [2008] Vol. 108, No. 1 (Part 3: October), S. 296. Auch Dennett bezieht sich auf solche Fälle der Fehleinschätzungen der eigenen Wahrnehmungsfähigkeit und auf hypothetische gegenteilige Fälle, bei denen er postuliert, dass sich solche Patienten über einen Verlust der Farbwahrnehmungsfähigkeit (cerebrale Achromatopsie bzw. cerebral achromatopsia) beklagen könnten, aber, wie er glaubt, es dennoch möglich sei, dass sie bei nicht-verbalen Farbtests immer noch gut abschneiden könnten. Vgl. Dennett, Daniel C.: »Quining Qualia«, in: Philosophy of mind. Classical and contemporary readings, Chalmers, David J. [Hrsg.], New York: Oxford University Press [zuerst 1988]/2002, S. 238-240.

81 So zeigt Metzinger u.a. auf, dass das ganzheitliche Ichgefühl sich unter experimentellen Bedingungen selektiv beeinflussen lässt. Versuchspersonen identifizierten sich beispielsweise aufgrund einer Manipulation mit einem computergenerierten Bild ihres eigenen Körpers. Vgl. Metzinger, Thomas: Der Ego Tunnel. Eine neue Philosophie des Selbst: Von der Hirnforschung zur Bewusstseinsethik, Berlin: Berliner Taschenbuch Verlag [zuerst 2009, engl.]/2011, S. 21. Im Zusammenhang mit dem »Ansturm« von neuem, empirischem Wissen über die »neuronale Maschinerie« folgert Metzinger denn auch, dass die »trügerische Selbstgewissheit der Introspektion [...] endgültig zerbrochen « sei. Metzinger, Thomas: Subjekt und Selbstmodell. Die Perspektivität phänomenalen Bewusstseins vor dem Hintergrund einer naturalistischen Theorie mentaler Repräsentation, Paderborn: Mentis 1999, S. 38 .

82 Vgl. Willaschek, Marcus: »Wahrnehmung VII (Analytische Philosophie)«, in: Historisches Wörterbuch der Philosophie. Band 12: W-Z, Ritter, Joachim / Kranz, Margarita [Hrsg.], Darmstadt: Wissenschaftliche Buchgesellschaft 1971-2007, S. 23 o.

83 A.a.O., S. 231. 
seien, wenn unsere Urteile bzw. Überzeugungen über sie falsch sein können. ${ }^{84}$ Doch erstens zeigt die Möglichkeit von falschen Urteilen über Qualia nicht auf, dass Qualia nicht als nicht-propositionale Erkenntnisformen epistemisch relevant sein können; zweitens sind Erkenntnisquellen, die einen bestimmten Fallibilitätsgrad aufweisen (welche Erkenntnisquelle hat dies nicht?) nicht per se epistemisch irrelevant und drittens besteht das hard problem bzw. QualiaProblem nicht darin, ob bspw. eine erlebte Emotion >veridisch veridisch ${ }^{85}$ ist, sondern darum, dass es irgendwie ist, eine Emotion zu erleben. Denn es ist auch irgendwie, eine Emotion zu erleben, wenn das Emotionserlebnis zu falliblen Urteilen über die Welt oder sogar über die Emotion selbst führt.

Trotz dieser ziemlich wackeligen Grundlage über die angebliche erkenntnistheoretische >Verzichtbarkeit $<$ wird, im Zusammenhang mit den oben erwähnten Fallibilitätsbeispielen, zuweilen ein weiterer Deutungssprung vollzogen, indem darüber spekuliert wird, ob wir uns nicht vielleicht in toto darüber täuschen, dass wir erlebende Kreaturen sind: [...] are you sure you're not a zombie? That you haven't been phenomenally pick-pocketed? That's just what a zombie would say!». ${ }^{86}$ Folgendes Argument, das auf der Prämisse unzutreffender Eigenschaften von Schwänen aufbaut, soll das Problem solcher Deutungssprünge sichtbar machen:

Prämisse 1: Wenn es Schwäne gibt, dann müssen sie die eigentümliche Eigenschaft haben, dass sie durchsichtige Lebewesen mit langem Hals sind, die sich nie täuschen. ${ }^{87}$

84 Vgl. dazu a.a.O., S. 231-232.

85 Ein Beispiel einer >nicht veridischen< Emotion wäre das Gefühl, dass jemand etwas gegen uns hat, obwohl das überhaupt nicht der Fall ist.

86 Mandik, Pete / Weisberg, Josh: »Type-q materialism«, 2008, Online: http://www. petemandik.com/philosophy/papers/typeq.pdf [zuletzt geprüft am 13.09.2018], S. 20 [Hervorhebung im Original]. Diese skeptische Hypothese begründet er folgendermassen: "If the notion of experience relevant is one wherein what is experienced is what is apparent, then what is apparent alone does not suffice for one to know, for instance, whether one has a red quale on the right side of one's own visual field. One needs, additionally, to rule out the skeptical hypothesis that maybe one's visual field houses unnoticed dancing qualia«. A.a.O., S. 15.

87 Analogie zur Prämisse 1: Qualia werden bspw. als intrinsisch, unmittelbar etc. definiert: "So when we look one last time at our original characterization of qualia, as ineffable, intrinsic, private, directly apprehensible properties of experience [...] «. Dennett, Daniel C.: »Quining Qualia«, in: Philosophy of mind. Classical and contemporary readings, Chalmers, David J. [Hrsg.], New York: Oxford University Press [zuerst 1988]/2002, S. 244. Oder Qualia sollen absolut infallibel (auch im Hinblick auf vergangene Urteile über Erleben) sein: »The infallibilist line on qualia treats them as properties of one's experience one cannot in principle misdiscover [...] «. A.a.O., S. 233. 
Prämisse 2: Es gibt keine durchsichtigen Lebewesen, die sich nie täuschen und einen langen Hals haben. ${ }^{8}$

Konklusion: Also gibt es auch keine Schwäne. ${ }^{89}$

Um von der richtigen Feststellung, dass unsere Urteile über gestrig Erlebtes (aufgrund der Interdependenz von kognitiven Prozessen und phänomenalem Erleben) falsch sein können, zu der Behauptung zu gelangen, dass wir >in Wirklichkeit< vielleicht alle Zombies sind, ist also noch ein gewaltiger metaphysischer Deutungssprung nötig. Die trügerische Rechtfertigung dieses Deutungssprungs liegt in einer, analog zur obigen >Prämisse $1<$ falschen, phänomenal inadäquaten Charakterisierung von Qualia.

Das Problem besteht also nicht nur in einem phänomenal inadäquaten Begriff über Qualia, der diesen eine spezifische Form vermeintlicher diachroner Infallibilität unterstellt, sondern zusätzlich darin, dass auf der Basis derart unzutreffender, den Qualia unterstellten Eigenschaften, ein infolgedessen auch unberechtigter metaphysischer Schluss gefällt wird, dass es kein phänomenales Erleben >gibt<. Dennoch lässt sich dieser Deutungssprung nicht nur bei Dennett ${ }^{90}$ beobachten, sondern bspw. auch bei Mandik, der noch

88 Analogie zur Prämisse 2: Die angeblichen Qualia-Eigenschaften werden widerlegt, indem bspw. mit den hypothetischen Kaffeetester Chase und Sanborn darauf hingewiesen wird, dass Geschmackserleben nicht >unmittelbar< und somit auch fallibel ist. Vgl. a.a.O., S. 231-236. Oder man beruft sich darauf, dass sich die angebliche Eigenschaft von Qualia als »phänomenale Atome« und die damit verbundene »Unteilbarkeits-Intuition« widerlegen lassen (so z.B. Metzinger, Thomas: Subjekt und Selbstmodell. Die Perspektivität phänomenalen Bewusstseins vor dem Hintergrund einer naturalistischen Theorie mentaler Repräsentation, Paderborn: Mentis 1999, S. 36.) bzw. dass die »Introspektion« generell »trügerisch« sei: so z.B. A.a.O., S. 38.

89 Analogie zur Konklusion: So zieht Dennett, unter dem Rückgriff auf seine QualiaDefinition (»[...] when we look one last time at our original characterization of qualia $[\ldots]$ «) den Schluss, dass es keine Qualia >gibt< $(»[\ldots]$ we find that there is nothing to fill the bill«). Dennett, Daniel C.: »Quining Qualia«, in: Philosophy of mind. Classical and contemporary readings, Chalmers, David J. [Hrsg.], New York: Oxford University Press [zuerst 1988]/2002, S. 244 [Hervorhebung E.E.]. Oder Mandik behauptet, dass wir nicht sicher sein können, ob wir nicht doch vielleicht Zombies sind, weil es ja möglich sein könnte, dass uns ein Qualia-Taschendieb, ohne dass wir dies gemerkt hätten, alle Qualia bereits geraubt haben könnte. Vgl. Mandik, Pete / Weisberg, Josh: »Type-q materialism «, 2008, Online: http://www.petemandik.com/philosophy/papers/typeq.pdf [zuletzt geprüft am 13.09.2018], S. 16 und 20.

90 Vgl. Dennett, Daniel C.: »Quining Qualia«, in: Philosophy of mind. Classical and contemporary readings, Chalmers, David J. [Hrsg.], New York: Oxford University Press [zuerst 1988]/2002, S. 229 und 244. 
expliziter die oben erwähnte, ziemlich seltsame These vertritt, dass wir uns vielleicht nur darüber täuschen, dass wir keine Zombies seien. ${ }^{91}$

Vielleicht liegt die Attraktivität einer Zuflucht in derlei ubiquitären Täuschungsthesen darin, dass damit ein schwieriges Explanandum, das einfach nicht so recht zum Naturalisierungsprogramm >passen< will, aus der Welt geschafft wäre: »It is hard to see how to find room in science for something that cannot be tested for or confirmed $\left[\ldots . .{ }^{9}{ }^{92}\right.$ So hofft und glaubt Mandik, dass Kinder, die in der Zukunft reduktiv-funktionalistische Konzeptionen des Phänomenalen lernen, dies ebenso >natürlich $<$ finden werden, wie die Theorie der Gravitation. ${ }^{93}$

\subsubsection{Nicht-Intentionalität}

Qualia haben nicht notwendigerweise die Eigenschaft der Nicht-Intentionalität. ${ }^{94}$ Phänomenales Erleben kann im Gegenteil intentionale Eigenschaften ${ }^{95}$ aufweisen: das Erleben unserer Furcht kann beispielsweise auf den Anblick eines gerade aus dem Käfig entwichenen Tigers gerichtet sein. ${ }^{96}$ Sonach gibt es Varianten von Intentionalität, die nicht von phänomenalem Erleben getrennt werden können. ${ }^{97}$ Das emotiv-somatische Erleben unsers Kummers kann auf

91 Vgl. Mandik, Pete / Weisberg, Josh: »Type-q materialism«, 20o8, Online: http://www. petemandik.com/philosophy/papers/typeq.pdf [zuletzt geprüft am 13.09.2018], S. 2 .

92 A.a.O., S. 2 o.

93 Vgl. a.a.O., S. 20.

94 Zur angeblichen Nicht-Intentionalität von Qualia vgl. z.B. Frank, Manfred: »Gibt es eine >innere Wahrnehmung«? «, in:Den eigenen Geist kennen. Selbstwissen, privilegierter Zugang und Autorität der ersten Person, Newen, Albert / Vosgerau, Gottfried [Hrsg.], Paderborn: Mentis 2005, S. 51. Oder: Metzinger, Thomas: Subjekt und Selbstmodell. Die Perspektivität phänomenalen Bewusstseins vor dem Hintergrund einer naturalistischen Theorie mentaler Repräsentation, Paderborn: Mentis 1999, S. 211. Vgl. ebenfalls Block, Ned J.: »Inverted Earth «, in: Philosophy of mind. Critical concepts in philosophy. Volume 4: Consciousness, Crawford, Sean [Hrsg.], London: Routledge \& Kegan Paul [zuerst 199o]/2011, S. 122 und 129.

95 Vgl. z.B. Goldie, Peter: The emotions. A philosophical exploration, New York: Oxford University Press 2002, S. 4, 54-55. Vgl. ebenfalls Graham, George / Horgan, Terence / Tienson, John: »Phenomenology, Intentionality, and the Unity of the Mind«, in: The Oxford Handbook of Philosophy of Mind, McLaughlin, Brian P. / Beckermann, Ansgar / Walter, Sven [Hrsg.], Oxford: Oxford University Press 2009, S. 530.

96 Vgl. dazu z.B. Pugmire, David: Rediscovering emotion, Edinburgh: Edinburgh University Press 1998, S. 3 o.

97 Vgl. Graham, George / Horgan, Terence / Tienson, John: »Phenomenology, Intentionality, and the Unity of the Mind «, in: The Oxford Handbook of Philosophy of Mind, McLaughlin, Brian P. / Beckermann, Ansgar / Walter, Sven [Hrsg.], Oxford: Oxford University Press 2009, S. 530. Vgl. dazu auch Goldie, Peter: The emotions. A philosophical exploration, New York: Oxford University Press 2002, S. 4, 54-55. 
die Person, die wir betrauern, gerichtet sein: »[...] our whole being aches in grief for the one we have lost«.98 Im Rahmen einer phänomenal adäquaten Erweiterung des Qualia-Begriffs um den Bereich der phänomenalen Intentionalität, bzw. I-Qualia, lässt sich unser Erleben nicht mehr auf die eingeengte Formel, nicht-intentionaler monosensualer Zustände beschränken, vielmehr kann sich phänomenales Erleben auch als eine »[...] bedeutungsvolle[n] Bezogenheit auf die Welt $[\ldots] \ll,{ }^{99}$ wie beispielsweise in Goldies Konzeption des »feeling towards $\aleph^{100}$ zeigen.

Deshalb ist es auch nicht gewinnbringend, Qualia auf vermeintlich nichtphänomenale, intentionale Gehalte reduzieren zu wollen. Und wenn Intentionalität selbst phänomenale Aspekte hat, dann ist der Versuch reduktionistischer Varianten repräsentationalistischer Theorien des Geistes, Phänomenalität auf Intentionalität und somit auf mit letzterer vermeintlich identischen Dritte-Person-Eigenschaften zu reduzieren (bzw. zu naturalisieren), ${ }^{101}$ nicht durchführbar. ${ }^{102}$

If phenomenology is partly constitutive of intentional content, as some philosophers suggest, then intentional properties may fail to supervene logically on the physical but they will supervene modulo conscious experience. ${ }^{103}$

So wird nach Engelen, aufgrund der Verflechtung des Emotionalen mit Intentionalität, sogar das mit Intentionalität fälschlicherweise in Verbindung

98 A.a.O., S. 55 [Hervorhebung im Original].

99 Wils, Jean-Pierre: »Sartre: Emotionen als Urteile«, in: Klassische Emotionstheorien. Von Platon bis Wittgenstein, Landweer, Hilge / Renz, Ursula [Hrsg.], Berlin: De Gruyter 2012, S. 666.

100 Goldie, Peter: The emotions. A philosophical exploration, New York: Oxford University Press 2002, S. 4.

101 Vgl. dazu Schildknecht, Christiane: »Phänomenales Erleben in Kunst und Literatur«, in: Kunst denken, Burri, Alex [Hrsg.], Paderborn: Mentis 2007, S. 97. Vgl. auch Graham, George / Horgan, Terence / Tienson, John: »Phenomenology, Intentionality, and the Unity of the Mind«, in: The Oxford Handbook of Philosophy of Mind, McLaughlin, Brian P. I Beckermann, Ansgar / Walter, Sven [Hrsg.], Oxford: Oxford University Press 20o9, S. 534.

102 Vgl. dazu Jacob, Pierre: »Intentionality«, in: The Stanford Encyclopedia of Philosophy, 2010, Online: http://plato.stanford.edu/archives/fall201o/entries/intentionality/ [zuletzt geprüft am 28.o9.2018], Abschnitt 10.o. Vgl. auch Searle, John R.: »Consciousness, Unconsciousness and Intentionality«, Philosophical Issues, [1991] Vol. 1: Consciousness, S. 48-49. Vgl. ebenfalls Graham, George / Horgan, Terence / Tienson, John: »Phenomenology, Intentionality, and the Unity of the Mind«, in: The Oxford Handbook of Philosophy of Mind, McLaughlin, Brian P. / Beckermann, Ansgar / Walter, Sven [Hrsg.], Oxford: Oxford University Press 20o9, S. 529 .

103 Chalmers, David J.: The conscious mind. In search of a fundamental theory, New York: Oxford University Press 1996, S. 82. 
gebrachte easy problem zu einem schwerwiegenden Problem. ${ }^{104}$ Mithin lassen sich phänomenales Erleben bzw. Qualia nicht mit intentionalen, angeblich nicht-phänomenalen Zuständen reduktiv erklären. Die Konsequenz eines erweiterten und phänomenal adäquaten Qualia-Begriffs, der die Phänomenalität der Intentionalität mit einschliesst, liegt demnach sogar in einer Verschärfung des hard problem. ${ }^{105}$

[...] the scope of the hard problem is much broader or more extensive than it is often said to be, for the expanded or whole hard problem incorporates the fact that the intentional, in a sense, is pervasively conscious. The hard problem is not limited to conscious states, in some narrowly parsed, intentionality-excluding way. The explanatory gap ramifies throughout the realm of the phenomenally intentional.106

\subsection{3 >Intrinsität, , Introspektivität< und >Innerlichkeit<}

Wie in Abschnitt 8.1 dargelegt, ist auch Intrinsität bzw. Introspektivität nicht notwendigerweise eine Qualia-Eigenschaft. Denn exterozeptive Sinneswahrnehmungen, die als gewöhnliche Standardfälle von Qualia gelten, müssen nicht erst >introspiziert oder durch eine ganz spezielle Art der >Introspektion< reflektiert werden, damit es irgendwie ist, das Gehörte oder Gesehene zu erleben. Damit es irgendwie ist, einen Flötenklang zu hören, müssen wir nur zuhören; und damit es irgendwie ist, ein Lichtspiel auf dem Meer zu sehen, müssen wir nur zusehen - um ein sinnlich-qualitatives Erlebnis durch unsere Exterozeption zu haben, benötigen wir gar keine Introspektion. ${ }^{107}$

Wenn Qualia überhaupt durch die so genannte >Introspektion< erlebt würden, dann handelte es sich paradoxerweise gerade um diejenigen phänomenalen Erlebnisse, die nicht im herkömmlichen monosensualen

104 Vgl. Engelen, Eva-Maria: »Zur Bedeutung von Sprache, Intentionalität und Erleben für das Verständnis von Emotionen«, in: Funktionen des Erlebens. Neue Perspektiven des qualitativen Bewusstseins. Band 5: Humanprojekt, Jung, Matthias / Heilinger, Jan-Christoph [Hrsg.], Berlin: De Gruyter 2009, S. 387 .

105 Vgl. dazu auch Pitt, David: »The Phenomenology of Cognition or what is it Like to Think that P? «, Philosophy and Phenomenological Research, [2004] Vol. 69, No. 1, S. 5 .

106 Graham, George / Horgan, Terence / Tienson, John: »Phenomenology, Intentionality, and the Unity of the Mind «, in: The Oxford Handbook of Philosophy of Mind, McLaughlin, Brian P. / Beckermann, Ansgar / Walter, Sven [Hrsg.], Oxford: Oxford University Press 2009, S. 534 [Hervorhebung E. E.].

107 Vgl. dazu auch Langsam, Harold L.: »Experiences, Thoughts, and Qualia«, Philosophical Studies, [2000] Vol. 99, No. 3, S. 269. Vgl. auch Beaton, Michael:»Qualia and Introspection«, Journal of Consciousness Studies, [2009] Vol. 16, No. 5, S. 105. Auch Stubenberg kritisiert die implizite Gleichsetzung von Introspektion mit phänomenalem Bewusstsein, vgl. weiter unten. Vgl. ebenfalls Stubenberg, Leopold: Consciousness and qualia, Amsterdam: John Benjamins 1998, S. 106. 
Qualia-Begriff enthalten sind, sondern um das mit der Interozeption und somatoviszeralen Zuständen zusammenhängenden Erleben von Emotionen. Da Emotionen, wie weiter unten dargelegt, in den wenigsten Fällen >unmittelbar< erlebt werden, sondern als interdependente Form emotiv-kognitiven bzw. emotiv-intentionalen Erlebens zu verstehen sind, handelt es sich bei ihnen aber auch nicht um eine rein sintrospektive< Art des Erlebens. Einzig (sinnesbasierte) Vorstellungen und der Ausschnitt somatischer Erlebnisformen, die unabhängig von Emotionen erlebt werden (wie das Empfinden von Schmerz, Kribbeln, Kitzeln etc.), könnte man folglich als genuin >introspektive< Erlebniszustände bezeichnen.

Hier sei also davor gewarnt, unserer vorschnellen, intuitiven Spaltung von >Aussenwelt< versus >Innenwelt< glauben zu schenken. Wie in Abschnitt 8.2.1 aufgezeigt wurde, besteht phänomenales Erleben nicht nur in introspektiven Imaginationen, wie bspw. der Phantasie einer roten Wolke, sondern auch in der (veridischen), exterozeptiven Wahrnehmung einer weissen Wolke. ${ }^{108} \mathrm{Zu}$ dem spielt es im Hinblick auf die Phänomenalität eines visuellen Erlebnisses gar keine Rolle, ob ein neuronaler Reiz bspw. aufgrund einer introspektiven Vorstellung gewissermassen >cortexintern< erfolgt oder wie bei einer exterozeptiven Farbwahrnehmung, seinen >Ursprung< in der Aussenwelt hatte. ${ }^{109}$ Aus diesen Gründen sei umso stärker zur Vorsicht geraten, dass wir nicht starre epistemische Bereiche von »intrinsisch «110 oder $»$ Innerlichkeit« ${ }^{111}$ bilden, in welchen Qualia Platz haben, gegenüber einem Bereich von diametral entgegengesetzten Wahrnehmungen der >Aussenwelt $<$, bei dem Qualia nicht in Betracht gezogen werden. ${ }^{112}$

Die dualistische Scheidung von >Introspektion< versus >Extrospektion< geht zurück auf eine Alltagsintuition, die durch den Eindruck geprägt ist, dass es so etwas wie abgrenzbare >homogene Einheiten` von Erleben gibt,

108 Vgl. dazu auch Langsam, Harold L.: »Experiences, Thoughts, and Qualia«, Philosophical Studies, [2000] Vol. 99, No. 3, S. 269. Vgl. dazu auch Beaton, Michael: »Qualia and Introspection «, Journal of Consciousness Studies, [2009] Vol. 16, No. 5, S. 105.

109 Vgl. dazu Roth, Gerhard: Das Gehirn und seine Wirklichkeit. Kognitive Neurobiologie und ihre philosophischen Konsequenzen, Frankfurt am Main: Suhrkamp 1994, S. 296.

110 Dennett, Daniel C.: »Quining Qualia «, in: Philosophy of mind. Classical and contemporary readings, Chalmers, David J. [Hrsg.], New York: Oxford University Press [zuerst 1988]/2002, S. 229.

111 Metzinger, Thomas: Subjekt und Selbstmodell. Die Perspektivität phänomenalen Bewusstseins vor dem Hintergrund einer naturalistischen Theorie mentaler Repräsentation, Paderborn: Mentis 1999, S. 37.

112 Vgl. Landgrebe, Ludwig / [Hrsg.: Novotný, Karel]: Der Begriff des Erlebens. Ein Beitrag zur Kritik unseres Selbstverständnisses und zum Problem der seelischen Ganzheit, Würzburg: Königshausen \& Neumann [zuerst 1932]/2010, S. 20 und 5o. 
die unsere Identität und unser >Innenleben< prägen. ${ }^{113}$ Auf der Basis dieser plakativen Unterscheidung entsteht dann eine Tendenz, qualitatives Erleben und das Phänomenale als reine »Innerlichkeit «114 von (erlebten) Eigenschaften der >Aussenwelt $<$ zu scheiden, obschon die >Aussenwelt $<$ (selbst wenn wir empirisch überprüfte Theorien über sie zu erwerben vermögen $)^{115}$ wahrnehmungstheoretisch auch nicht anders, als durch sinnliche Phänomenalität erlebt werden kann. So stellt Roth fest, dass unsere Empfindung, dass wahrgenommene Dinge »ausserhalb «116 sind, »nur innerhalb «117 derjenigen »Wirklichkeit«118 existiert, die als »phänomenale Welt «119 ein Konstrukt des Gehirns darstellt. ${ }^{120}$ Die dualistische Scheidung zwischen >Introspektion< versus $>$ Extrospektion<, die quer durch bewusstes Erleben gehen soll, ist daher auch in epistemischer und neuropsychologischer Hinsicht problematisch. ${ }^{121}$ Der Versuch, bewusstem Erleben seine Relevanz als Explanandum abzusprechen, indem es einfach als eine >eigentümliche Innerlichkeit gedeutet wird, scheitert deshalb am Desiderat phänomenaler Adäquatheit. ${ }^{122}$ Denn auch dieses vermeintlich von Phänomenalität vollständig getrennte >Aussen<, d.h. unsere Umwelt, erleben wir je subjektiv. ${ }^{123}$ Die mit phänomenaler Inadäquatheit

113 Vgl. a.a.O., S. 20.

114 Metzinger, Thomas: Subjekt und Selbstmodell. Die Perspektivität phänomenalen Bewusstseins vor dem Hintergrund einer naturalistischen Theorie mentaler Repräsentation, Paderborn: Mentis 1999, S. 37 .

115 Freilich baut unser theoretisches Wissen von der Aussenwelt nicht nur auf dem Erleben dieser Welt auf, sondern auch auf darauf aufbauenden und wiederum durch Empirie geprüften Theorien.

116 Roth, Gerhard: Das Gehirn und seine Wirklichkeit. Kognitive Neurobiologie und ihre philosophischen Konsequenzen, Frankfurt am Main: Suhrkamp 1994, S. 325.

117 Ebd.

118 Ebd.

119 A.a.O., S. 324.

120 Das ist aber nicht so zu verstehen, dass Roth damit behauptet, es gäbe keine »reale« bzw. »bewusstseinsjenseitige« Welt - diese ist uns jedoch Roth zufolge nicht zugänglich. Vgl. a.a.O., S. 325. Auch behauptet er nicht, wie Eliminativisten das tun würden, dass die Konstruktion der Wirklichkeit bzw. der »phänomenale[n] Welt« nicht >existiert<. Vgl. a.a.O., S. 324. Vgl. dazu auch a.a.O., S. 324-326 und 332-333.

121 Vgl. dazu auch a.a.O., S. 296 und 320-321. Vgl. auch Langsam, Harold L.: »Experiences, Thoughts, and Qualia«, Philosophical Studies, [200o] Vol. 99, No. 3, S. 269. Vgl. ebenfalls Landgrebe, Ludwig / [Hrsg.: Novotný, Karel]: Der Begriff des Erlebens. Ein Beitrag zur Kritik unseres Selbstverständnisses und zum Problem der seelischen Ganzheit, Würzburg: Königshausen \& Neumann [zuerst 1932]/2010, S. 20 und 5 o.

122 Vgl. dazu Stubenberg, Leopold: Consciousness and qualia, Amsterdam: John Benjamins 1998, S. 278 .

123 Phänomenales Erleben ist aber auch nicht etwas, das als externe Eigenschaft von Objektoberflächen, gleichsam >draussen herumliegt<, sich wie Pilze aufsammeln und nach 
verbundene Reduzierung von bewusstem Erleben oder sogar von Bewusstsein auf Introspektion nennt Stubenberg »the introspective access assumption«. ${ }^{124}$ Mit ihm lässt sich denn auch die Frage stellen, warum sich phänomenales Erleben nur auf Introspektion beschränken soll. Stubenberg stellt sogar die Frage, warum man überhaupt Introspektion mit Qualia in Verbindung bringt:

At this point we are addressing the question why anybody would think that introspection has something to do with qualitative consciousness. Or, put more strongly, we are asking why anybody would think that it is obvious that introspection has everything to do with consciousness. ${ }^{125}$

Doch sowohl die Churchlands ${ }^{126}$ als auch Dennett ${ }^{127}$ nehmen in ihren Versuchen, Qualia zu eliminieren, Zuflucht auf diese kontroverse und angebliche Qualia-Eigenschaft der >Introspektion< bzw. einer damit assoziierten >Intrinsität ${ }^{128}$ Dennetts Versuche, letztere angebliche Eigenschaften von Qualia zu widerlegen, bestehen im Wesentlichen darin, wieder auf die erwähnten Schwierigkeiten des Gedankenexperiments mit den beiden Kaffeetestern Chase und Sanborn (bzw. mit einem Gedankenexperiment zu einer

seinen >Merkmalen $<$ beschreiben lässt. Wenn wir fragen, wo wir diese phänomenalen Erlebnisse finden, so sind wir freilich je einzeln auf uns selbst verwiesen. Diese Verwiesenheit ist jedoch sowohl bei sintrinsischen< (bzw. introspektiven und enterozeptiven) als auch >empirischen< (bzw. exterozeptiven) Fällen des Erlebens vorhanden. Vgl. dazu auch Landgrebe, Ludwig / [Hrsg.: Novotný, Karel]: Der Begriff des Erlebens. Ein Beitrag zur Kritik unseres Selbstverständnisses und zum Problem der seelischen Ganzheit, Würzburg: Königshausen \& Neumann [zuerst 1932]/2010, S. 19.

124 Stubenberg beschreibt diese (von ihm kritisierte) Ansicht der »introspective access assumption«, bei der man davon ausgeht, dass nur durch Introspektion phänomenale Zustände bewusst werden können, im Detail folgendermassen: »Introspection is the only means by which the mind can present qualitative states to itself so as to make them into possible objects of belief or knowledge. Thus introspecting one's qualitative states becomes a necessary condition on one's cognizing those states. [...] The state's being introspected brings it within the purview of our propositional attitudes. As long as the qualitative state exists unintrospected it is nothing for the subject «. Stubenberg, Leopold: Consciousness and qualia, Amsterdam: John Benjamins 1998, S. 106 [Hervorhebung im Original].

125 A.a.O., S. $105^{-106 .}$

126 Vgl. Churchland, Paul M.: Matter and consciousness. A contemporary introduction to the philosophy of mind, Cambridge, Massachusetts: MIT Press 1988.

127 Vgl. dazu auch Dennett, Daniel C.: »Quining Qualia«, in: Philosophy of mind. Classical and contemporary readings, Chalmers, David J. [Hrsg.], New York: Oxford University Press [zuerst 1988]/2002, S. 238-241.

128 Vgl.dazuauch Ramsey,William:»Eliminative Materialism«,in:TheStanfordEncyclopedia of Philosophy, 2013, Online: http://plato.stanford.edu/archives/sum2013/entries/materialismeliminative/ [zuletzt geprüft am 25.09.2018], Abschnitt 3.1. 
Gesichtsfeld-Inversion ${ }^{129}$ oder über hypothetische Reaktionen auf Blumenkohlgeschmack $)^{130}$ zu verweisen: »[...] the considerations raised by Chase's quandary show that it is far from obvious that any intrinsic property [...] could play the role of anchor for the Lockean relational treatment of the public perceptual properties «. ${ }^{131} \mathrm{Im}$ Gedankenexperiment über Reaktionsmöglichkeiten auf Blumenkohlgeschmack geht es, analog zur >Intuitionspumpe< mit den Kaffeetestern, darum, dass es schwierig wäre, festzustellen, ob sich durch eine geschmacksverändernde Pille, die (mentale?) Reaktion auf den Geschmack von Blumenkohl oder das phänomenale Erleben des Blumenkohlgeschmacks selbst verändert hat. Damit glaubt Dennett, nachzuweisen, dass das Erleben des Blumenkohlgeschmackes keine anderen Eigenschaften habe, ausser dispositionalen, reaktionserzeugenden Eigenschaften. ${ }^{132}$

Diese Beispiele zeigen aber, analog zu seinem Argument gegen die angebliche diachrone Infallibilität von Qualia, wiederum nur auf, dass phänomenales Erleben nicht notwendigerweise >unmittelbar ist und auch nicht unabhängig von kognitiven Prozessen stattfindet. Doch bei einem phänomenal adäquaten Qualia Begriff, der die Interdependenz von kognitiven, emotionalen und somatischen Prozessen mitberücksichtigt, und deshalb emotive Qualia und >emotiv-kognitives Erleben< zu integrieren vermag, ist sowohl das sinnlichqualitative Erleben der Umwelt als auch das somatosensorisch-qualitative Erleben des eigenen Körpers fast nie unmittelbar. So zeigen die Fälle von $E$ Qualia und EK-Qualia auf, dass das somatosensorische Erleben des eigenen Körpers und das Erleben der Umwelt als Formen der Wahrnehmung ${ }^{133}$ betrachtet werden müssen, die durch Urteile, »Affektlogiken ${ }^{134}{ }^{134}$ emotionale

129 Mit dieser »Intuitionspumpe« verweist Dennett darauf, dass es möglich sei, sich an bestimmte Eigenheiten, wie eine Gesichtsfeldinversion, zu gewöhnen. Versuchspersonen hätten sich nach dem mehrtägigen Tragen von Gesichtsfeldinversions-Brillen an diese eigentümliche Art, zu sehen (funktional) gut angepasst. Aus dem Gewöhnungseffekt an die Gesichtsfeldinversion schliesst Dennett, dass es deshalb keine »intrinsische Verkehrtheit« der Wahrnehmung geben könne. Vgl. dazu Dennett, Daniel C.: »Quining Qualia«, in: Philosophy of mind. Classical and contemporary readings, Chalmers, David J. [Hrsg.], New York: Oxford University Press [zuerst 1988]/2002, S. 238.

13 V Vgl. dazu a.a.O., S. 237-238.

131 A.a.O., S. 241.

132 Vgl. dazu a.a.O., S. 237-238.

133 Vgl. Prinz, Jesse: »Are Emotions Feelings? «, Journal of Consciousness Studies, [2005] Vol. 12, No. 8-10, S. 23.

134 Ciompi, Luc: Die emotionalen Grundlagen des Denkens. Entwurf einer fraktalen Affektlogik, Göttingen: Vandenhoeck \& Ruprecht 1997, S. 45. 
Gedanken «,135 Situationsbewertungen ${ }^{136}$ und durch einen intentionalen Bezug auf das Objekt der Emotion ${ }^{137}$ stark >gefärbt< und mithin fast nie >unmittelbar sind. ${ }^{138}$ Aufgrund der Interdependenz von kognitiven, emotionalen und somatischen Erlebnisprozessen sind deshalb umgekehrt auch unsere Kognition und die mit ihnen verbundenen Situationsbewertungen nicht zwingend isoliert oder gar >unmittelbar, sondern wir denken und fühlen (und handeln), wenn wir beispielsweise eine länger andauernde Emotion der Wut erleben, in »wütenden Arten und Weisen «, 139 weil Emotionen eben zugleich kognitive Weisen »of minding things «140 sind. Das bedeutet, dass unter der Berücksichtigung von EK-Qualia auch unsere Urteile und Gedanken, weil sie durch Stimmungen und Emotionen >gefärbt $<$ werden, nicht >reine $<$ statische und intrinsisch-unmittelbar< erlebte Kognitionen, sondern vielmehr in einer dynamisch-heterogenen Weise (als emotiv-kognitive Gehalte) erlebt werden.

Wenn der Kaffeetester Chase, den Kaffeegeschmack heute, nachdem seine Frau verstorben ist, als >bitterer< erlebt als vor einem Jahr, heisst das also nur, dass phänomenales Erleben nicht notwendigerweise >intrinsisch-unmittelbar<, d.h. unabhängig von emotiv-kognitiven Prozessen stattfindet. Erlebt Chase deswegen überhaupt keinen Kaffeegeschmack? Und gibt es, nur weil Chase die angebliche »Selbigkeit« ${ }^{141}$ und angebliche sintrinsische Unmittelbarkeit< von Kaffeegeschmack selbstverständlich nicht diachron identifizieren kann weil diese »Selbigkeit«142 im realen, dynamisch-heterogenen Erleben kaum je vorkommt - deshalb kein dynamisches, mit emotiv-kognitiven Erlebniszuständen interagierendes Kaffeegeschmacks-Erleben?

135 Stocker, Michael: »Emotional Thoughts«, American Philosophical Quarterly, [1987] Vol. 24, No. 1, S. 6o.

136 Vgl. Damasio, Antonio R.: Descartes' error. Emotion, reason and the human brain, London: Vintage [zuerst 1994]/2006, S. 146 und 224-225. Vgl. ebenfalls Damasio, Antonio R.: Looking for Spinoza. Joy, sorrow and the feeling brain, London: Vintage 2004, S. 89.

137 Vgl. z.B. Goldie, Peter: The emotions. A philosophical exploration, New York: Oxford University Press 2002, S. 15-18 und 54-55.

138 Vgl. z.B. Ciompi, Luc: Die emotionalen Grundlagen des Denkens. Entwurf einer fraktalen Affektlogik, Göttingen: Vandenhoeck \& Ruprecht 1997, S. 96. Vgl. ebenfalls Pugmire, David: Rediscovering emotion, Edinburgh: Edinburgh University Press 1998, S. 75.

139 Stocker, Michael: »Einige Betrachtungen zu intellektuellen Wünschen und Emotionen«, in: Philosophie der Gefühle, Döring, Sabine [Hrsg.], Frankfurt am Main: Suhrkamp [zuerst 2004, engl.]/2009, S. 214.

140 Pugmire, David: Rediscovering emotion, Edinburgh: Edinburgh University Press 1998, S. 4 [Hervorhebung im Original].

141 Metzinger, Thomas [Hrsg.]: Grundkurs Philosophie des Geistes. Band 1: Phänomenales Bewusstsein, Paderborn: Mentis 2007, S. 203.

142 Ebd. 
Eine weitere Variante, phänomenalem Erleben seine Relevanz in epistemischer Hinsicht abzusprechen oder anzuzweifeln, besteht darin, es auf eine »introspizierende Alltagspsychologie $«^{143}$ zu reduzieren. Mit dieser Reduktion bzw. epistemischen Abwertung von phänomenalem Erleben wird dann die Behauptung verbunden, (der Einbezug von) Phänomenalität verkörpere einen gänzlich anderen »epistemischen Zugang « ${ }^{144} \mathrm{zu}$ den Problemen der Bewusstseinsphilosophie als der intersubjektive, empirisch-naturalistische. So stellt Metzinger phänomenales Erleben als »systeminterne Selbstwahrnehmung«145 bzw. ausschliessliche $» I n n e r l i c h k e i{ }^{146}$ der Intersubjektivität wissenschaftlicher Theorienkonstruktion gegenüber:

Dieser theoretische Zugang [die empirische Psychologie] wird niemals dieselbe Innerlichkeit erzeugen können wie die systeminterne Selbstwahrnehmung, denn im Gegenteil ist das epistemische Ziel wissenschaftlicher Theorienkonstruktion ja gerade Intersubjektivität. ${ }^{147}$

Von einer ähnlich homogen-introspektiven Einheit von Erleben, das angeblich nur unser >Innenleben< betrifft, scheint auch Dennett auszugehen, wenn er behauptet, dass Qualia den letzten »Verteidigungsgraben der Innerlichkeit« (»ditch defense of the inwardness «) $)^{148}$ des Mentalen darstellen. Doch erstens beschränkt sich das Spektrum phänomenalen Erlebens, wie oben aufgezeigt, nicht auf eine »systeminterne Selbstwahrnehmung « ${ }^{149}$ bzw. ausschliessliche »Innerlichkeit «. ${ }^{150}$ Derphänomenale Charakter bewussten Erlebens ist zweitens nicht nur durch eine einzige »introspizierende «151 Perspektive eines einzelnen Subjektes, sondern immer auch für andere Subjekte epistemisch - und insofern intersubjektiv - zugänglich. Denn wenn Phänomenalität durch zahllose Individuen erlebt wurde und wird, so handelt es sich um phänomenologische

143 Metzinger, Thomas: Subjekt und Selbstmodell. Die Perspektivität phänomenalen Bewusstseins vor dem Hintergrund einer naturalistischen Theorie mentaler Repräsentation, Paderborn: Mentis 1999, S. 37 .

144 Ebd.

145 Ebd.

146 Ebd.

147 Ebd. [Ergänzung in Klammern von E.E.].

148 Dennett, Daniel C.: »Quining Qualia «, in: Philosophy of mind. Classical and contemporary readings, Chalmers, David J. [Hrsg.], New York: Oxford University Press [zuerst 1988]/2002, S. 229.

149 Metzinger, Thomas: Subjekt und Selbstmodell. Die Perspektivität phänomenalen Bewusstseins vor dem Hintergrund einer naturalistischen Theorie mentaler Repräsentation, Paderborn: Mentis 1999, S. 37 .

150 Ebd.

151 Ebd. 
Daten, die (wenn es sich nicht um Randphänomene wahnhaften Erlebens handelt) intersubjektiv nachprüfbar sind.

An isolated phenomenological report can be discounted with impunity. But to disregard or contradict a widespread phenomenological report is no more reasonable than to suppress available but uncomfortable experimental results. ${ }^{152}$

Wenn das Erleben von Emotionen oder von Schmerz intersubjektiv insoweit geteilt werden kann, als durch deren Beschreibung die subjektiven Räumlichkeiten von zwei oder mehr Personen zur Deckung kommen, dann ist es nicht plausibel, von einer epistemisch in der Subjektivität >gefangenen< und >isolierten< Phänomenalität zu sprechen. ${ }^{153}$ Der fälschlicherweise als blosse »Innerlichkeit « 154 abgewertete Einbezug phänomenaler Daten weist demnach vielmehr eine intersubjektive Dimension, analog zur Intersubjektivität der empirischen Basis wissenschaftlicher Theorienkonstruktionen auf. Denn letztere ist fundamental durch Sinneserlebnisse strukturiert: unser Weltbezug und unsere Theorienkonstruktionen, hätten keinerlei Basis, die systematisch gedeutet könnten, wenn wir Taubblind wären und zudem keine taktilen Organe zum Ertasten von Gegenständen hätten. Anders gesagt: gäbe es keinerlei bewusstes Welt-Erleben, gäbe es auch nichts Empirisches, das systematisch, bspw. durch wissenschaftliche Theorienkonstruktionen gedeutet werden könnte.

Wissenschaft findet nicht unabhängig von Bewusstsein, gewissermassen in einer Faktenwelt von >objektiven<, bewusstseinunabhängigen Gegenständen statt, sondern auch auf der Grundlage von beobachtenden, bewussten

152 Stubenberg, Leopold: Consciousness and qualia, Amsterdam: John Benjamins 1998, S. 118 [Hervorhebung E. E.].

153 Vgl. dazu auch Fuchs, Thomas: »Hirnwelt oder Lebenswelt? Zur Kritik des Neurokonstruktivismus«, Deutsche Zeitschrift für Philosophie, [2011] Vol. 59, No. 3, S. 351,354 und 356. So ist auch das Erleben von Emotionen nach Pugmire nicht etwas, das nur durch Introspektion zugänglich ist, sondern auch durch deren Charakterisierung mit Hilfe von Beschreibungen. Pugmire, David: Rediscovering emotion, Edinburgh: Edinburgh University Press 1998, S. 96. Vgl. dazu auch Damasio, Antonio R.: The feeling of what happens. Body, emotion and the making of consciousness, London: Vintage 2000, S. 309. Zum Intersubjektiven Erleben von Qualia vgl. auch Schildknecht, Christiane: »Phänomenales Erleben in Kunst und Literatur«, in: Kunst denken, Burri, Alex [Hrsg.], Paderborn: Mentis 2007, S. 103 .

154 Metzinger, Thomas: Subjekt und Selbstmodell. Die Perspektivität phänomenalen Bewusstseins vor dem Hintergrund einer naturalistischen Theorie mentaler Repräsentation, Paderborn: Mentis 1999, S. 37. 
Subjekten, die durch Inter-Subjektivität verbunden sind. ${ }^{155}$ Ja, sogar die Bedingung der Möglichkeit, dass es so etwas wie Naturwissenschaften geben kann, besteht darin, dass sie geistige Hervorbringungen des Menschen sind. ${ }^{156}$ Intersubjektivität basiert epistemisch auf zwar zusammenarbeitenden, aber letztlich doch je einzelnen, erlebenden Subjekten - auch wenn noch so viele Subjekte die Evidenz der als sintersubjektiv geltenden Tatsachen stützen mögen: »[...] Erste-Person-Daten der direkten Erfahrung [sind] in der letzten epistemologischen Analyse die Bestätigungsbasis aller Arten tatsachenbezogener Wissensbehauptungen «. ${ }^{157}$ Auch das begriffliche System der Physik ist in sensorischen Beobachtungen verankert, ${ }^{158}$ und selbst ein/e Neurowissenschafter/in kann die Korrelate des Erlebens einer Versuchsperson nur auf der Grundlage ihres eigenen bewussten Erlebens beobachten:»[...] observations are also experiences $[\ldots] \ll . .159$

So gesteht Gerhard Roth ein, dass sich auch die Hirnforschung nur innerhalb derjenigen »Wirklichkeit«, ${ }^{160}$ die er mit einer »phänomenale[n] Welt «161 in Verbindung bringt, vollzieht. ${ }^{162}$ Hirnforschung kann demzufolge, nach Roths Terminologie, nur »wirkliche Gehirne«163 der »phänomenale[n] Welt «, 164 aber »niemals reale «165 Gehirne bzw. solche einer »bewusstseinsjenseitige[n] Welt«166 untersuchen. Denn das »reale Gehirn«167 sei uns

155 Vgl. dazu auch Husserl, Edmund: Cartesianische Meditationen. Eine Einleitung in die Phänomenologie, Hamburg: Meiner [zuerst 1955]/1995, S. 85 .

${ }_{15} 6$ Vgl. dazu auch Schildknecht, Christiane: »Der Dualismus und die Rettung der Phänomene«, in: Homo Sapiens und Homo Faber. Epistemische und technische Rationalität in Antike und Gegenwart. Festschrift für Jürgen Mittelstrass, Wolters, Gereon / Mittelstrass, Jürgen [Hrsg.], Berlin: W. de Gruyter 2005, S. 265.

157 Feigl, Herbert: »Das >Mentale und das >Physikalische««, in: Grundkurs Philosophie des Geistes. Band 2: Das Leib-Seele-Problem, Metzinger, Thomas [Hrsg.], Paderborn: Mentis [zuerst 1958, engl.]/2007, S. 151 [Hervorhebung im Original; Ergänzung in Klammern von E. E.].

$15^{8}$ Vgl. dazu a.a.O., S. 159 .

159 Velmans, Max: »Reflexive Monism. Psychophysical Relations among Mind, Matter, and Consciousness«, Journal of Consciousness Studies, [2012] Vol. 19, No. 9-10, S. 148.

160 Roth, Gerhard: Das Gehirn und seine Wirklichkeit. Kognitive Neurobiologie und ihre philosophischen Konsequenzen, Frankfurt am Main: Suhrkamp 1994, S. 324.

161 Ebd.

162 Vgl. a.a.O., S. 333.

163 Ebd.

164 A.a.O., S. 324.

165 A.a.O., S. 333 .

166 A.a.O., S. 325 .

167 A.a.O., S. $33^{2}$. 
»unzugänglich «. ${ }^{168}$ Mit anderen Worten: auch ein Gehirn ist uns epistemisch nie wirklich >objektiv< zugänglich. Die Intuition, dass wir einen >objektiven< Zugang zu Gehirnen haben, könnte ihre Ursache in der Verwechslung unserer Konzeption bewusstseinsunabhängiger Gegenstände mit unseren visuellen und taktilen Sinnes-Erlebnissen haben. Diese Verwechslung könnte in uns den Eindruck entstehen lassen, dass das gesehene (oder betastete) Hirn identisch mit unserer Konzeption eines >bewusstseinsunabhängigen Hirnes< ist. Denn gerade aufgrund unserer Sinneserlebnisse sind wir in der Lage, auf Gegenstände zu schliessen, von denen wir dann eine bewusstseinsunabhängige Variation des Gesehenen konzeptualisieren.

When this disturbance reaches the brain, the physiologist has the experience which is called sseeing the other man's brain<. [...] The relation of a visual experience to the physical object that common sense thinks it is seeing is thus indirect and causal, and there is no reason to suppose that close similarity between them that common sense imagines to exist. ${ }^{169}$

Einerseits bilden bewusste (Sinnes-) Erlebnisse also eine unverzichtbare Grundlage von Empirie. Andererseits kann Phänomenalität, insbesondere auch deshalb, weil bewusstes Erleben ubiquitär ist, nicht von Empirie getrennt werden. Wenn Erleben aber, wie behauptet, ausschliesslich in einer seltsamen »Innerlichkeit«170 und Introspektivität bestehen würde, so wären auch die Erlebnisse einer Neurowissenschafterin etwas Seltsames. Denn wenn man dem Erleben und damit verbundenen Wahrnehmungen nicht vertrauen könnte, dann könnte man Velmans zufolge, weil es sich bei Beobachtungen einer Neurowissenschafterin ebenfalls um bewusstes Erleben handelt, auch nicht länger den empirischen Wissenschaften vertrauen. ${ }^{171}$

168 Ebd. Diese Feststellung sollte aber nach Roth nicht als »[...] eine Aussage über die tatsächliche Beschaffenheit der Realität missverstanden werden [...] «. A.a.O., S. 325.

169 Russell, Bertrand: »Excerpt from >Mind and matter««, in: Philosophy of mind. Critical concepts in philosophy. Volume 1: Foundations, Crawford, Sean [Hrsg.], London: Routledge \& Kegan Paul [zuerst 1956]/2011, S. 173.

170 Metzinger, Thomas: Subjekt und Selbstmodell. Die Perspektivität phänomenalen Bewusstseins vor dem Hintergrund einer naturalistischen Theorie mentaler Repräsentation, Paderborn: Mentis 1999, S. 37 .

171 So konstatiert Velmans: »If S's >experiences< [die Erlebnisse einer Versuchsperson] of the world cannot be trusted because they are private and subjective, neither can E's >observations< [die Beobachtungs-Erlebnisse einer Wissenschafterin] of the world, which have a phenomenology that is equally private and subjective to $\mathrm{E}$. But if $\mathrm{E}$ cannot trust observed or experienced phenomena as primary evidence of whatever is taking place, he can no longer trust empirical science $[. .$.$] «. Velmans, Max: »Reflexive$ 
Aufgrund der vermeintlich gegensätzlichen epistemischen Bereiche von »Innerlichkeit «, ${ }^{172}$ in welcher Qualia Platz haben, gegenüber angeblich nichtphänomenalen Wahrnehmungen der >Aussenwelt $<,{ }^{173}$ glaubt man jedoch, Phänomenalität der Intersubjektivität gegenüberstellen zu können. Nun ist aber nicht einmal das, was wir >Intersubjektivität< nennen, etwas >Objektives<, sondern, wie wir mit Damasio behaupten können, eher eine kollektive Subjektivität mit interpersonaler Ähnlichkeit:

[...] since you and I are similar enough biologically to construct a similar enough image of the same thing, we can accept without protest the conventional idea that we have formed the picture of some particular thing. But we did not. ${ }^{174}$

\subsubsection{Homogenität und >Atomizität}

Im Zusammenhang mit der oben aufgeführten, diachronen Fallibilität von Qualia zieht bspw. Metzinger in Erwägung, dass wir Erlebnisinhalte, wenn sie »einfache ${ }^{175}$ Inhalte seien, nicht nur von anderen aktuellen Inhalten unterscheiden können müssen, sondern dass wir ihre »Selbigkeit«176 auch diachron feststellen können sollten. Dies sei deshalb nötig, weil wir sonst einen Erlebnisgehalt nicht einem phänomenologischen Begriff zuordnen könnten. Und diese Reidentifikation sei wiederum die Voraussetzung, um Qualia, im Sinne von Dennett, als »essentials«177 bzw. als »einfachen phänomenalen Inhalt«, 178 einen ontologischen Status zuweisen zu können. ${ }^{179}$

Monism. Psychophysical Relations among Mind, Matter, and Consciousness«, Journal of Consciousness Studies, [2012] Vol. 19, No. 9-10, S. 149 [Ergänzung in Klammern von E.E.].

172 Dennett, Daniel C.: »Quining Qualia«, in: Philosophy of mind. Classical and contemporary readings, Chalmers, David J. [Hrsg.], New York: Oxford University Press [zuerst 1988]/2002, S. 229.

173 Vgl. dazu auch Sokolowski, Robert: Introduction to phenomenology, Cambridge, Massachusetts: Cambridge University Press 2007, S. 12.

174 Damasio, Antonio R.: The feeling of what happens. Body, emotion and the making of consciousness, London: Vintage 2000, S. 321 [Hervorhebung im Original]. Vgl. auch a.a.O., S. 309 und 320.

175 Metzinger, Thomas [Hrsg.]: Grundkurs Philosophie des Geistes. Band 1: Phänomenales Bewusstsein, Paderborn: Mentis 2007, S. 203.

176 Ebd.

177 Dennett, Daniel C.: »Quining Qualia«, in: Philosophy of mind. Classical and contemporary readings, Chalmers, David J. [Hrsg.], New York: Oxford University Press [zuerst 1988]/2002, S. 228.

178 Metzinger, Thomas [Hrsg.]: Grundkurs Philosophie des Geistes. Band 1: Phänomenales Bewusstsein, Paderborn: Mentis 2007, S. 203.

179 Vgl. dazu auch a.a.O., S. 204. 
Was aber, wenn Qualia nicht als »homogen[e] $\ll^{180}$ Monaden $^{181}$ und nicht als »Atome des Bewusstseins « 182 zu verstehen sind? Denn jene monadischen Vorstellungen von Erleben entsprechen einem viel zu engen, statischen und monosensualen Verständnis, ${ }^{183}$ welches der Komplexität, dem Verlaufscharakter und dem Aspekt der Gesamtheit heterogener Erlebnis-Nuancen eines phänomenal adäquaten Qualia-Begriffs nicht standhalten. ${ }^{184}$ So ist nach Stubenberg ein Rot-Erlebnis nicht etwas Homogen-Isoliertes, sondern ein Teil eines »Bündels«, ${ }^{185}$ der die phänomenalen Qualitäten konstituiert, die zusammen mit vielen andern Erlebnis-Nuancen die Heterogenität der Phänomenalität ausmachen. ${ }^{186}$

Wenn also bspw. Metzinger auf der Grundlage solch einer monadischen und verengten Qualia-Konzeptionen fragt: »[...] gibt es eigentlich >den< einfachen phänomenalen Inhalt? «, ${ }^{187}$ dann muss man mit einem phänomenal adäquaten Qualia-Begriff antworten: >Nein, es gibt diesen >einfachen Gehalt allenfalls als Idealtyp - qualitatives Erleben zeigt sich, phänomenal adäquat betrachtet, vielmehr als komplexe, heterogene und sich (u.a. auch durch somatisch-emotive und emotiv-kognitive Prozesse) ständig ändernde Form des Erlebens im Sinne eines reichhaltigen Qualia-Spektrums $\longleftarrow{ }^{188}$ Eine solche Antwort zeigt gleichzeitig auf, dass das Explanandum des hard problem nicht mit verengten Qualia-Begriffen und mit Hinweisen auf dessen angebliche Marginalität, Eigentümlichkeit, Atomizität, Innerlichkeit, Intrinsität vor-

180 A.a.O., S. 203.

181 Zur monadischen Konzeption von Qualia vgl. auch Lycan, William: »Representational Theories of Consciousness«, in: The Stanford Encyclopedia of Philosophy, 2008, Online: http://plato.stanford.edu/archives/fall2oo8/entries/consciousness-representational/ [zuletzt geprüft am 28.o9.2018], Abschnitt 1.o. Vgl. ebenfalls Metzinger, Thomas: Subjekt und Selbstmodell. Die Perspektivität phänomenalen Bewusstseins vor dem Hintergrund einer naturalistischen Theorie mentaler Repräsentation, Paderborn: Mentis 1999, S. 33.

182 Metzinger, Thomas [Hrsg.]: Grundkurs Philosophie des Geistes. Band 1: Phänomenales Bewusstsein, Paderborn: Mentis 2007, S. 58.

183 Zum monosensualen Verständnis von Qualia vgl.: Lorenz, Kuno: Artikel »Qualia« in: Enzyklopädie Philosophie und Wissenschaftstheorie, [Hrsg.: Mittelstrass, Jürgen], Stuttgart / Weimar: J. B. Metzler Verlag 2004, Band 3, S. 426-427.

184 Vgl. dazu auch Jung, Matthias / Heilinger, Jan-Christoph [Hrsg.]: Funktionen des Erlebens. Neue Perspektiven des qualitativen Bewusstseins. Band 5: Humanprojekt, Berlin: De Gruyter 20o9, S. 10.

185 Stubenberg, Leopold: Consciousness and qualia, Amsterdam: John Benjamins 1998, S. 288.

186 Vgl. ebd.

187 Metzinger, Thomas [Hrsg.]: Grundkurs Philosophie des Geistes. Band 1: Phänomenales Bewusstsein, Paderborn: Mentis 2007, S. 203 [Hervorhebung im Original]. Vgl. ebenfalls a.a.O., S. $57-58$.

188 Kein Zitat, sondern eine fiktive Antwort von EE. 
schnell aus dem Weg geräumt werden kann, sondern dass die Reichhaltigkeit und Ubiquität von phänomenalem Erleben dieses Problem im Gegenteil noch viel schwieriger machen.

\subsection{Immense Vielfalt und Ubiquität von Qualia}

\subsubsection{Reichhaltigkeit phänomenalen Erlebens - das Qualia-Spektrum}

Mit einem phänomenal adäquaten Qualia-Begriff wird sichtbar, dass es einer Transformation des Explanandums gleichkommt, wenn bewusstes Erleben auf >isolierte $<$ monosensuale, monadische Sinnesgehalte wie beispielsweise Farboder Geschmacksempfindungen der Einfachheit halber eingeengt werden oder in angeblich homogene und »einfache phänomenalen Inhalt $[\mathrm{e}] \ll^{189}$ aufgespalten werden. ${ }^{190}$ Selbst wenn man die oben erwähnten kognitiven Formen phänomenalen Erlebens ausser Acht lässt, können wir feststellen, dass wir fast nie homogene und monosensuale Qualia erleben. So lassen sich beispielsweise Kombinationen von Geruchs- und Geschmacksempfindungen, die mit einem Lustempfinden interagieren oder die vielschichtigen auditiven Qualia eines Musikstückes, das mit einem intensiven emotionalen Erleben verwoben sind, nur künstlich voneinander trennen. ${ }^{191}$

Dann aber erweist sich der bisherige Qualia-Begriff als zu eng gefasst und es ist phänomenologisch und systematisch adäquat, analog zu Stubenberg, von »Qualia-Bündeln«192 auszugehen, die innerhalb des phänomenalen Raumes eines »Qualia-Spektrums «193 erfahren werden. Die Stärke des Ansatzes von Stubenberg liegt nicht zuletzt darin, dass ihm zufolge Qualia bzw. QualiaBündel nicht eine geheimnisvolle, tiefere Realität sind, sondern den Teil der Realität bilden, welcher für uns überhaupt zugänglich ist - zudem konstituieren

189 A.a.O., S. 203.Vgl. dazu ebenfalls a.a.O., S. 57. Weitere Beispiele atomistisch-monosensualer Verwendungsweisen des Qualia-Begriffs mit homogenen Eigenschaften finden sich bei Robinson, William S.: »Qualia realism «, in: A Field Guide to the Philosophy of Mind, SIFA, Società Italiana Filosofia Analitica, 2002, Online: http://host.uniroma3.it/progetti/kant/ field/qr.htm [zuletzt geprüft am 25.09.2018], Abschnitt einleitender Erläuterungen.

190 Vgl. dazu auch Stubenberg, Leopold: Consciousness and qualia, Amsterdam: John Benjamins 1998, S. 33 .

191 Vgl. dazu Chalmers, David J.: The conscious mind. In search of a fundamental theory, New York: Oxford University Press 1996, S. 10.

192 Stubenberg, Leopold: Consciousness and qualia, Amsterdam: John Benjamins 1998, S. 286, 288 und 310 .

193 Heckmann, Heinz-Dieter: »Qualia-basierte Argumente gegen den Materialismus«, in: Bewusstsein und Repräsentation, Esken, Frank / Heckmann, Heinz-Dieter [Hrsg.], Paderborn: Mentis 1999, S. 331. 
sie unser Bewusstsein: »Yoo are a bundle of percepts (among other things) and the tomato percept is a member of this bundle. [...] to be conscious is to have qualia $[\ldots] \ll .194$ Eine derartige Qualia-Konzeption hat neben ihrer phänomenalen Adäquatheit den Vorteil, dass sie nicht auf jenen seltsamen und problematischen ontologischen Entitäten gründet, die Qualia-Kritiker zu Recht für einen Atomismus des Bewusstseins verantwortlich machen. ${ }^{195}$

Ignorierte man das reichhaltige und heterogene Spektrum des Erlebens, hätten phänomenale Realisten tatsächlich ein Problem mit jenen eigenartigen »Atomen des Bewusstseins «. ${ }^{196}$ Deshalb konzipiert Stubenberg Qualia, im Gegensatz zu Dennett, ${ }^{197}$ nicht als etwas Essenzielles oder als ein Substrat, sondern als heterogenes Konglomerat phänomenaler Gehalte, die unser Erleben konstituieren: »[...] the phenomenal red is a member of the bundle of compresent phenomenal qualities that together constitute the tomato as I experience it $\ll .198$

Wenn man dem Desiderat phänomenaler Adäquatheit standhält und die komplexe Vielfalt phänomenalen Erlebens anerkennt, ${ }^{199}$ die sich aus der Berücksichtigung von Erlebnisqualitäten der Propriozeption, der Viszerozeption, des Gleichgewichts- und Temperatursinns, der Mechanorezeption, der >emotiven Qualia< bzw. E-Qualia, der EK-Qualia (>emotiv-kognitives Erleben), der I-Qualia (phänomenale Intentionalität) und nicht zuletzt aller Variationen und Kombinationen auditiver, ${ }^{200}$ olfaktorischer, ${ }^{201}$ gustatorischer oder visueller Sinnesmodalitäten ergibt, dann kann man mit Pitt feststellen, dass die phänomenale Vielfalt schier unendlich wird. Dies trifft erst recht zu, wenn

194 Stubenberg, Leopold: Consciousness and qualia, Amsterdam: John Benjamins 1998, S. 310.

195 Vgl. dazu Metzinger, Thomas [Hrsg.]: Grundkurs Philosophie des Geistes. Band 1: Phänomenales Bewusstsein, Paderborn: Mentis 2007, S. 57-58 und 203.

196 A.a.O., S. 58.

197 Vgl. Dennett, Daniel C.: »Quining Qualia«, in: Philosophy of mind. Classical and contemporary readings, Chalmers, David J. [Hrsg.], New York: Oxford University Press [zuerst 1988]/2002, S. 228.

198 Stubenberg, Leopold: Consciousness and qualia, Amsterdam: John Benjamins 1998, S. 288. Vgl. dazu auch a.a.O., S. 285-288 und 308-310.

199 Vgl. dazu auch Van Gulick, Robert: »Consciousness«, in: The Stanford Encyclopedia of Philosophy, 2014, Online: http://plato.stanford.edu/archives/spr2014/entries/ consciousness/ [zuletzt geprüft am 17.09.2018], Abschnitt 2.2.

200 Vgl. z.B. Chalmers, David J.: The conscious mind. In search of a fundamental theory, New York: Oxford University Press 1996, S. 7.

201 Vgl z.B. Roth, Gerhard: Das Gehirn und seine Wirklichkeit. Kognitive Neurobiologie und ihre philosophischen Konsequenzen, Frankfurt am Main: Suhrkamp 1994, S. 22 und 104. Vgl. ebenfalls Lorenz, Kuno: »Qualia«, in: Enzyklopädie Philosophie und Wissenschaftstheorie. Band 3: P-So, Mittelstrass, Jürgen [Hrsg.], Stuttgart: Metzler 2004, S. 426. 
wir auch noch die Phänomenalität von nicht-emotiven Denk-Qualia bzw. D-Qualia berücksichtigen.

I also do not think it is especially implausible to claim a distinctive phenomenology for each of the indefinitely many thoughts we are capable of consciously entertaining - because, in particular, there are just too many of them. Consider, for example, how many distinct visual scenes we are capable of distinguishing an astronomically large number, I would say. The productive capacity of the phenomenal mind is prodigious. ${ }^{202}$

Nicht zuletzt legen neuropsychologische Überlegungen von Roth nahe, dass unser Bewusstsein immer von einem heterogenen Spektrum diverser mentaler Erlebnisse umgeben ist, die»[...] injedem Augenblickinganz unterschiedlichen Mischungsverhältnissen $\left[\ldots . . \ll^{203}\right.$ vorliegen. Roth nennt diese phänomenale Heterogenität »[...] den charakteristischen vielfarbigen Strom meiner bewussten Existenz « ${ }^{204}$ und Slaby spricht bezüglich der Mannigfaltigkeit unseres stetigen (Hintergrund-) Empfindens von einem »polydimensionalen Empfinden «. ${ }^{205}$ Schliesslich können wir mit Heckmann feststellen, »[...] dass unser mentales Leben ein für Menschen typisches Qualia-Spektrum hat, das den Kernbestand menschlicher Subjektivität ausmacht «. ${ }^{206}$

Die hier aufgeführten Schilderungen eines phänomenal adäquaten QualiaBegriffs zeigen auf, dass man deshalb von einem äusserst reichhaltigen `Qualia-Spektrumく sprechen muss, weil phänomenales Erleben sich weder auf die komplexe Heterogenität aller Sinnesmodalitäten noch auf emotive oder emotiv-intentionale Erlebnisgehalte beschränkt, sondern auch noch den nahezu unendlich viele phänomenale Möglichkeiten umfassenden Bereich bewusst erlebter Gedanken, Verstehenserlebnisse, Fantasien und Vorstellungen etc. beinhaltet:

The spectrum of conscious experience, as Galen Strawson remarks (1994:4), ranges from $>$ the most purely sensory experiences to the most abstractly cognitive experiences $<$. Theses last include $>$ the experience of consciously entertained

202 Pitt, David: »The Phenomenology of Cognition or what is it Like to Think that P?«, Philosophy andPhenomenologicalResearch, [2004] Vol.69, No.1, S. ${ }_{2}$ [Hervorhebung E. E.].

203 Roth, Gerhard: Das Gehirn und seine Wirklichkeit. Kognitive Neurobiologie und ihre philosophischen Konsequenzen, Frankfurt am Main: Suhrkamp 1994, S. 273.

204 Ebd.

205 Slaby, Jan: Gefühl und Weltbezug. Die menschliche Affektivität im Kontext einer neoexistentialistischen Konzeption von Personalität, Paderborn: Mentis 2008, S. 109.

206 Heckmann, Heinz-Dieter: »Qualia-basierte Argumente gegen den Materialismus«, in: Bewusstsein und Repräsentation, Esken, Frank / Heckmann, Heinz-Dieter [Hrsg.], Paderborn: Mentis 1999, S. 331. 
thought, of reading and understanding, of unplanned fantasy, and of directed imagining $<^{207}$

Wenn selbst konzeptuelle bzw. gedankliche Zustände phänomenale Aspekte haben, dann stellt dies Pitt zufolge ein zusätzliches Problem für reduktionistische Ansätze dar. ${ }^{208} \mathrm{Ja}$, wenn bewusst erlebte Gedanken, ihre je eigene Phänomenalität aufweisen, dann muss es doch sogar für Eliminativisten irgendwie sein, wenn sie gerade wieder einmal denken, dass es kein Erleben $>$ gibt $<.209$

\subsubsection{Qualia als ubiquitäres Bewusstseinsphänomen}

$[\ldots]$ the sea of qualia $[\ldots]$ is much, much deeper than suspected. [...] Once one appreciates the full scope of qualia, the claim that it would be like nothing to live a life without them will, I hope, be more plausible. >The life of the mind $<$ is parasitic upon the >internal illumination. If the inner light goes out you are, in Einstein's words, nothing but a pile of dirt. ${ }^{210}$

Die in Abschnitt 9.3.1 dargelegte, schier unendliche Vielfalt und Polydimensionalität phänomenalen Erlebens zeigt ebenfalls auf, dass wir mit einem phänomenal adäquaten Qualia-Begriff davon ausgehen müssen, dass

207 Graham, George / Horgan, Terence / Tienson, John: »Phenomenology, Intentionality, and the Unity of the Mind«, in: The Oxford Handbook of Philosophy of Mind, McLaughlin, Brian P. / Beckermann, Ansgar / Walter, Sven [Hrsg.], Oxford: Oxford University Press 2009, S. 517.

208 Vgl. dazu Pitt, der die Frage über die Phänomenalität von gedanklichen Zuständen an jener Stelle jedoch offen lässt. Pitt, David: »Mental Representation«, in: The Stanford Encyclopedia of Philosophy, 2008, Online: http://plato.stanford.edu/archives/fall2008/ entries/mental-representation [zuletzt geprüft am 25.09.2018], Abschnitt 3.o. An anderer Stelle spricht er sich jedoch klar für die Phänomenalität von Gedanken aus, vgl. Pitt, David: »The Phenomenology of Cognition or what is it Like to Think that P?«, Philosophy and Phenomenological Research, [2004] Vol. 69, No. 1, S. 2.

209 Etwas genauer lässt sich dieses Beispiel so umschreiben: Wenn jemand denkt: >Qualia existieren nicht! Dies lässt sich nicht mit einem naturwissenschaftlichen Weltbild unter einen Hut bringen!, so wird diese Person, wenn es sich bei ihr um einen bewussten Gedanken handeln soll, diesen Denkakt kognitiv erleben (D-Qualia). Der qualitative Erlebnisgehalt, wie es ist, diesen Gedanken zu denken, kann - wenn sich diese Person zudem noch über die >Qualia-Freaks` aufregt - freilich auch einen emotiven Aspekt (EKQualia) und einen intentionalen Aspekt (I-Qualia) aufweisen. Vgl. dazu auch Graham, George / Horgan, Terence / Tienson, John: »Phenomenology, Intentionality, and the Unity of the Mind «, in: The Oxford Handbook of Philosophy of Mind, McLaughlin, Brian P. I Beckermann, Ansgar / Walter, Sven [Hrsg.], Oxford: Oxford University Press 2009, S. 517.

210 Stubenberg, Leopold: Consciousness and qualia, Amsterdam: John Benjamins 1998, S. $25^{-26 .}$ 
Phänomenalität ein ubiquitäres Bewusstseinsphänomen ist: »[...] it is not just information that is ubiquitous. Experience is ubiquitous too «. ${ }^{211}$ Es geht uns, wie Slaby feststellt, zu jeder Zeit »irgendwie «. ${ }^{212}$ Das bedeutet, dass mit eliminativistischen (bzw. phänomenal antirealistischen) ${ }^{213}$ und phänomenal indifferenten ${ }^{214}$ Theorien des Geistes, sämtliche Aspekte mentaler Zustände entweder vernachlässigt oder geleugnet werden, sofern es sich nicht um unbewusste Zustände handelt. ${ }^{215}$ Daraus ergibt sich die paradoxe Situation, dass wir es bei solchen Ansätzen mit >Theorien des Geistes< zu tun haben, deren Explananda nichts mit denjenigen mentalen Phänomenen zu tun haben, die bewusste Organismen wirklich auszeichnen. So stellt Strawson fest, dass die Leugnung von Erleben, das Seltsamste sei, was in der Geschichte menschlichen Denkens je gedacht wurde.

>They are prepared to deny the existence of experience.< At this we should stop and wonder. I think we should feel very sober, and a little afraid, at the power of human credulity, the capacity of human minds to be gripped by theory, by faith. For this particular denial is the strangest thing that has ever happened in the whole history of human thought, not just the whole history of philosophy. ${ }^{216}$

Welchen (substanz-) ontologischen Status bewusstes Erleben und Phänomenales haben, ist deshalb, im Hinblick auf dessen zentralen Stellenwert als Explanandum, eher zweitrangig. Aufgrund des engen Zusammenhangs zwischen dem hard problem, Bewusstsein und phänomenalem Erleben ist es aber unverzichtbar, Qualia hinreichend als Explanandum zu berücksichtigen und näher als üblich zu bestimmen. Wenn Qualia ein ubiquitäres Bewusstseinphänomen sind, dann ist deren Berücksichtigung und die damit verbundene exakte Bestimmung dieses Explanandums eine Voraussetzung dafür, ob es bei einer Theorie des Geistes überhaupt um das geht, was bewusste Organismen auszeichnet. Wenn eine Theorie des Geistes sich mit dem hard problem ernsthaft auseinander setzen will, dann ist deshalb eine hinreichende und phänomenal adäquate Beachtung von Qualia bzw. bewusstem Erleben

211 Chalmers, David J:: The conscious mind. In search of a fundamental theory, New York: Oxford University Press 1996, S. 293. Vgl. dazu ebenfalls a.a.O., S. 297 und 298.

212 Slaby, Jan: Gefühl und Weltbezug. Die menschliche Affektivität im Kontext einer neoexistentialistischen Konzeption von Personalität, Paderborn: Mentis 2008, S. 15 o.

213 Vgl. dazu Abschnitt 7.1.

214 Vgl. dazu Abschnitt 7.2.

215 Vgl. dazu auch Stubenberg, Leopold: Consciousness and qualia, Amsterdam: John Benjamins 1998, S. 3.

216 Strawson, Galen: »Realistic Monism. Why Physicalism Entails Panpsychism«, Journal of Consciousness Studies, [2006] Vol. 13, No. 10-11, S. 5 . 
unumgänglich. ${ }^{217}$ Deshalb ist eine Konzeption des Mentalen, ohne einen Einbezug von bewusstem Erleben nicht hinreichend, sofern sie nicht nur eine Theorie über funktionale Prozesse (in Zombies) sein soll.

You can talk about various other features of consciousness - for example, the powers that the visual system has to discriminate colors [...]. But you can't set qualia on one side, because if you do there is no consciousness left over. ${ }^{218}$

Weil Erleben nur mit Bewusstsein möglich ist, ist das hard problem auch das Problem des Bewusstseins: »The problem of explaining these phenomenal qualities is just the problem of explaining consciousness. This is the really hard part of the mind-body problem «. ${ }^{219}$ So können wir mit Chalmers und Searle das hard problem, das Qualia-Problem und das Problem des Bewusstseins als gleichbedeutend erachten:»[...] the problem of qualia is not just an aspect of the problem of consciousness; it is the problem of consciousness « ${ }^{220}$

Auch die in dieser Arbeit beschriebene und durch einen phänomenal adäquaten Qualia-Begriff sichtbar werdende Ubiquität von Qualia weist darauf hin, dass bewusstes Erleben und Phänomenalität nicht voneinander getrennt werden können. Bewusstsein und Qualia sind so stark verwoben, dass Searle gemäss alle bewussten Phänomene Qualia sind. ${ }^{221}$ So betrachtet, besteht Bewusstsein in einer Serie phänomenaler Zustände, weil jeder bewusste $\mathrm{Zu}$ stand phänomenal ist:

[...] of course, all conscious phenomena are qualitative, subjective experiences, and hence are qualia. There are not two types of phenomena, consciousness and qualia. There is just consciousness, which is a series of qualitative states. ${ }^{222}$

217 Bewusstes Erleben, ja Bewusstsein überhaupt ist, wie sich weiter unten zeigen wird, eng verwoben, wenn nicht identisch mit phänomenalem Erleben. Vgl. dazu Vgl. dazu auch McGinn, Colin: »Can We Solve the Mind-Body Problem?«, in: Mind, New Series, [1989] Vol. 98, No. 391, S. 349. Vgl. ebenfalls Jung, Matthias / Heilinger, Jan-Christoph [Hrsg.]: Funktionen des Erlebens. Neue Perspektiven des qualitativen Bewusstseins. Band 5: Humanprojekt, Berlin: De Gruyter 2009, S. 7. Vgl. dazu auch Chalmers, David J.: The conscious mind. In search of a fundamental theory, New York: Oxford University Press 1996, S. 6.

218 Searle, John R.: The mystery of consciousness, New York: New York Review Books 1997, S. 28 [Hervorhebung E. E.].

219 Chalmers, David J:: The conscious mind. In search of a fundamental theory, New York: Oxford University Press 1996, S. 4.

220 Searle, John R.: The mystery of consciousness, New York: New York Review Books 1997, S. 28 [Hervorhebung im Original].

221 Vgl. dazu a.a.O., S. 9 und 5 o.

222 A.a.O., S. 9. 
Weil es immer irgendwie ist, einen bewussten mentalen Zustand $\mathrm{zu}$ erleben, ist bewusstes Erleben immer auch phänomenales Erleben. Bewusstsein kann demnach als die Totalität von phänomenalem Erleben verstanden werden: »Consciousness, then, is the totality of our qualia «. ${ }^{223}$ Insofern kann der Qualia-Begriff auch irreführend sein: »[...] every conscious state is a qualitative state, and >qualia< is just a misleading name for the consciousness of all conscious states «. ${ }^{224}$ Umso irreführender ist ein Qualia-Begriff, der durch eine phänomenal inadäquate bzw. transformierende Einschränkung die angebliche >Marginalität< von Qualia implizieren will.

Wenn jedoch Qualia, auf der Grundlage der Berücksichtigung ihrer Ubiquität, ihrem diachronen Verlaufscharakter und ihrer schier unendlichen Vielfalt phänomenal adäquat konzipiert werden, so können wir mit Stubenberg Qualia und Bewusstsein als interdependente Phänomene betrachten - ja Qualia sind, aufgrund ihrer Ubiquität das, was unser Sein bei Bewusstsein ausmacht:

[...] qualia are the phenomenal properties we meet with every moment of our waking and dreaming lives. [...] we are immersed in a sea of qualia, [...] we are subject to a ceaseless barrage of qualia, $[\ldots]$ we are inundated with qualia, $[\ldots]$ we are swamped by qualia, [...] we find ourselves thrown into an ocean of qualia. [...] Having qualia is what being conscious consists in. That is, having qualia must account for the fact that it is like something to be us. ${ }^{225}$

Die Ubiquität phänomenalen Erlebens hat nicht zuletzt zur Folge, dass Phänomenalität, bewusstes Erleben, Bewusstsein ${ }^{226}$ und Qualia sogar als identische Phänomene verstanden werden können: » $[. .$.$] to be conscious is to$ have qualia $[. ..] \ll{ }^{227}$ Ohne Bewusstsein ist phänomenales Erleben nicht möglich; und umgekehrt bezeichnen wir einen Organismus als >Bewusstlos $<$, wenn er nichts erlebt. Eine notwendige Eigenschaft, die Qualia besitzen, ist deshalb

223 Elitzur, Avshalom C.:»Consciousness makes a difference: A reluctant dualist's confession«, 2009, Online: http://philpapers.org/archive/ELICMA.pdf [zuletzt geprüft am 13.09.2018], S. 10.

224 Searle, John R.: The mystery of consciousness, New York: New York Review Books 1997, S. 5 .

225 Stubenberg, Leopold: Consciousness and qualia, Amsterdam: John Benjamins 1998, S. 33 [Hervorhebung E. E.].

226 Im Zusammenhang mit dem hier aufgeführten erweiterten Qualia-Begriff spielt die Unterscheidung zwischen angeblich möglichen phänomenalen Zuständen, die sunbewusst< (funktional nicht verfügbar) sind, und solchen, die bewusst sind, keine Rolle. Da >unbewusste< Phänomenalität, auch keine für diese Arbeit relevante Form bewussten Erlebens darstellt. Vgl. dazu auch Block, Ned J.: »On a confusion about a function of consciousness «, Behavioral and Brain Sciences, [1995] Vol. 18, No. 2, S. 231-233.

227 Stubenberg, Leopold: Consciousness and qualia, Amsterdam: John Benjamins 1998, S. 310. 
deren Bewusstheit oder das mit ihnen verbundene Sein-bei-Bewusstsein. Qualia können folglich nur als bewusste mentale Zustände vorkommen:

[...] qualia are necessarily conscious, i.e., that they can be instantiated only in conscious events. This conclusion makes qualia eligible as types of consciousness, and provides a possible answer to the question $>$ What is the difference between conscious events and nonconscious events? « namely, the answer, that conscious events are those that instantiate qualia. ${ }^{228}$

Eine weitere notwendige Eigenschaft von Qualia, die mit deren Eigenschaft der Bewusstheit zusammenhängt, besteht darin, dass es irgendwie ist, sie zu erleben. Um das hard problem anzugehen, gibt es deshalb sowohl kein Vorbeikommen am Phänomen >Bewusstsein $<$ als auch am Phänomen >Erleben<. Auch wenn es aus Gründen der ontologischen Sparsamkeit einfacher wäre, Bewusstsein und Phänomenalität zu ignorieren oder zu bestreiten, so sind die Kosten, die durch den oben beschriebenen phänomenalen Antirealismus bzw. durch phänomenale Indifferenz anfallen, unter Einbezug der Unentwirrbarkeit von phänomenalem Erleben und Bewusstsein noch höher. Das ist deshalb der Fall, weil der damit einhergehende Verlust explanatorischer Adäquatheit noch grösser wird. Die explanatorische Inadäquatheit hat seine Ursache nämlich im mit solchen Positionen zwingend einhergehenden Ignorieren von der vermutlich zentralsten Eigenschaft des Mentalen: Bewusstsein.

Aus diesem Grund ist auch für den reduktiven Funktionalismus die notorische Unterbestimmung von phänomenalen Bewusstseinszuständen ein Problem, weil er letztere nur durch kausale und funktionale Rollen wie Inputs, Outputs und anderer interner Zustande zu bestimmen vermag. ${ }^{229}$ Obwohl die reduktive Variante des Funktionalismus phänomenale Bewusstseinszustände nicht (explizit) leugnet, gehören diese, wie erwähnt, schon aus methodischen Gründen gar nicht zu den möglichen Gegenständen einer funktionalen Erklärung von Erleben. ${ }^{230}$ Um aber konstatieren zu können, dass Erleben zwar

228 Robinson, William S.: »Qualia realism «, in: A Field Guide to the Philosophy of Mind, SIFA, Società Italiana Filosofia Analitica, 2002, Online: http://host.uniromaz.it/progetti/kant/ field/qr.htm [zuletzt geprüft am 25.09.2018], Abschnitt »Three Further Properties« [Hervorhebung im Original].

229 Vgl. z.B. Pauen, Michael: Das Rätsel des Bewusstseins. Eine Erklärungsstrategie, Paderborn: Mentis 2001, S. 174. Vgl. ebenfalls Armstrong, David M.: A materialist theory of the mind, London: Routledge \& Kegan Paul 1968. Vgl. auch Armstrong, David M.: The nature of mind, Brighton, Sussex: The Harvester Press 1980. Vgl. ebenfalls Lewis, David: »Reduction of Mind«, in: Papers in metaphysics and epistemology, Lewis, David [Hrsg.], Cambridge, Massachusetts: Cambridge University Press 1994, S. 291-324.

230 Vgl. Pauen, Michael: Das Rätsel des Bewusstseins. Eine Erklärungsstrategie, Paderborn: Mentis 2001, S. 197. 
existiert, esaber $>$ eigentlich $\measuredangle n u r a u s k a u s a l e n u n d f u n k t i o n a l e n R o l l e n b e s t e h t,{ }^{231}$ muss er das Phänomen >Erleben< implizit voraussetzen. Ein analoges Problem hat der Repräsentationalismus: so ist nach Levine die Idee, Bewusstsein in eine (meta-) repräsentationale Relation einbauen zu können, eine zirkuläre Konzeption. ${ }^{232}$ Weder HOT- noch HOP-Theorien der Metarepräsentation können die Kluft, die zwischen dem subjektiven Phänomen `Erleben< und einer >objektiven< (Meta-) Repräsentation besteht, plausibel überbrücken. ${ }^{233}$ Das Ausklammern von Bewusstsein ist daher die Ursache dafür, dass sich mit demjenigen Explanans, mit dem versucht wurde, phänomenale auf nichtphänomenale Eigenschaften zu reduzieren, kein sinnvoller Bezug mehr zum Explanandum herstellen lässt: Warum soll eine Repräsentation, gegenüber anderen Repräsentationen, zu einem bewussten Erlebnis mutieren, nur weil es sich bei ihr um eine höherstufige Repräsentation handelt? Warum und wie sollen Bewusstheit und Phänomenalität entstehen, wenn eine Repräsentation eine andere Repräsentation repräsentiert? Warum soll eine Repräsentation zweiter Stufe, nur weil sie eine Repräsentation erster Stufe repräsentiert, und mithin etwas komplizierter als jene offenbar >bewusstlose stufige Repräsentationen ist, plötzlich die Eigenschaft von Bewusstheit und Phänomenalität gewinnen?

Wenn Phänomenalität und Bewusstsein schon in der Fragestellung zum hard problem vernachlässigt, umgedeutet oder ausgeklammert werden, dann resultiert das in einer schwerwiegenden Problematik: Dem Explanans (also z.B. einer Repräsentation, einem neuronalen Korrelat oder einer Funktion) fehlt leider ausgerechnet diejenige Eigenschaft, die mit ihr ja hätte erklärt werden sollen. Die >Erklärung`von Phänomenalität durch ein Explanans, dem man eigentlich nur >objektive< Dritte-Person-Eigenschaften zuschreiben kann, scheint dann eben nur noch möglich, indem man Phänomenalität nicht mehr als etwas zu Erklärendes betrachtet, sondern - ohne weiter darauf

231 Vgl. Schröder, Jürgen: »Qualia und Physikalismus«, Journal for General Philosophy of Science, [1997] Vol. 28, No. 1, S. 172.

232 Vgl. Levine, Joseph: »The Explanatory Gap«, in: The Oxford Handbook of Philosophy of Mind, McLaughlin, Brian P. / Beckermann, Ansgar / Walter, Sven [Hrsg.], Oxford: Oxford University Press 2009, S. 288.

233 Vgl. dazu auch Stubenberg, Leopold: Consciousness and qualia, Amsterdam: John Benjamins 1998, S. 6 o. 
einzugehen - implizit als gegeben voraussetzt ${ }^{234}$ oder sie entweder halbherzig ${ }^{235}$ oder vollständig ${ }^{236}$ leugnet.

Im Zusammenhang mit der in dieser Arbeit ausgewiesenen Ubiquität von Qualia und des unentwirrbaren Zusammenhangs zwischen Qualia und Bewusstsein gilt es, mit Searle vor einer gleichsam >dualistischen< Konzeption von Bewusstsein - die dem Explanandum des hard problem auszuweichen versucht - zu warnen. Denn wir sollten uns auch nicht davon trügen lassen, dass wir das harte Problem der Qualia einfach ignorieren können, indem wir uns vom Konstrukt leiten lassen, dass es zwei >Arten< von Bewusstsein geben könnte: ein informationsverarbeitendes Bewusstsein, das für die Wissenschaft zugänglich sei und ein eigenartiges, mysteriöses phänomenales Bewusstsein, welches man als vermeintlich »marginales « ${ }^{237}$ Phänomen ruhig ausklammern können soll. Nein, eine Spaltung des Bewusstseins in Teile, die man ausklammert und andere, die man als objektiv zugängliches Explanandum anerkennt, verletzt unsere Forderung einer phänomenal adäquaten Behandlung von Bewusstsein fundamental:

No, the unity of consciousness guarantees that for each of us all the variety of the forms of our conscious life are unified into a single conscious field. This includes bodily sensations [...] seeing a rose; and it also includes thinking about mathematical problems or the next election. ${ }^{238}$

234 So muss, wie oben kritisiert, bspw. der reduktive Funktionalismus das Phänomen >Erleben<, um es reduktiv-funktional >erklärenく zu können, im Explanans bereits voraussetzen. Vgl. dazu z.B. Pauen, Michael:Das Rätsel des Bewusstseins. Eine Erklärungsstrategie, Paderborn: Mentis 2001, S. 197.

235 So geht der externalistische Repräsentationalismus davon aus, dass bei normaler veridischer Wahrnehmung die erlebten phänomenalen Eigenschaften »nicht wirklich « existieren, sondern Eigenschaften geistunabhängiger Objekte seien. Vgl. dazu Tye, Michael: »Qualia«, in: The Stanford Encyclopedia of Philosophy, 2007, Online: http://plato. stanford.edu/archives/fall2007/entries/qualia/ [zuletzt geprüft am 25.09.2018], Abschnitt 6.o. Vgl. ebenfalls Dretske, Fred: »Woher wissen Sie, dass Sie kein Zombie sind?«, in: Den eigenen Geist kennen. Selbstwissen, privilegierter Zugang und Autorität der ersten Person, Newen, Albert / Vosgerau, Gottfried [Hrsg.], Paderborn: Mentis [zuerst 2003, engl.]/2005, S. 102.

236 Zur vollständigen Leugnung von Phänomenalität im Eliminativismus vgl. z.B. Dennett, Daniel C.: »Quining Qualia«, in: Philosophy of mind. Classical and contemporary readings, Chalmers, David J. [Hrsg.], New York: Oxford University Press [zuerst 1988]/2002, S. 229.

237 Churchland, Paul M.: »The Rediscovery of Light«, The Journal of Philosophy, [1996] Vol. 93, No. 5 , S. 223 .

238 Searle, John R.: The mystery of consciousness, New York: New York Review Books 1997, S. 200. 